\title{
The Lead-Glass Electromagnetic Calorimeter for the SELEX Experiment
}

\author{
M. Y. Balatz ${ }^{\mathrm{a}, 1}$, P. S. Cooper ${ }^{\mathrm{b}, 2}$, G. V. Davidenko $^{\mathrm{a}}$,
} A. G. Dolgolenko ${ }^{\text {a }}$, G. B. Dzyubenko ${ }^{\text {a }}$, A. V. Evdokimov ${ }^{\mathrm{a}, 3}$, I. Giller ${ }^{\text {c }}$, Y. M. Goncharenko ${ }^{\text {d }}$, A. D. Kamenskii ${ }^{\mathrm{a}}$, M. A. Kubantsev ${ }^{\mathrm{a}, 4}$, V. F. Kurshetsov ${ }^{\mathrm{d}}$, J. Lach ${ }^{\mathrm{b}}$, L. G. Landsberg ${ }^{\text {d }}$, I. F. Larin ${ }^{\text {a }}$, V. A. Matveev ${ }^{\mathrm{a}}$, M. A. Moinester ${ }^{c}$, S. B. Nurushev ${ }^{\text {d }}$, A. Ocherashvili ${ }^{c}$, J. Russ ${ }^{\text {e,2 }}$, V. K. Semyatchkin ${ }^{\text {a }}$, A. I. Sitnikov ${ }^{\text {a }}$, V. Steiner ${ }^{c}$,

I. I. Tsukerman ${ }^{\text {a }}$, A. N. Vasiliev ${ }^{\mathrm{d}}$, D. V. Vavilov ${ }^{\mathrm{d}}$, V. S. Verebryusov ${ }^{a}$, V. A. Victorov ${ }^{d}$, V. E. Vishnyakov ${ }^{a}$.

${ }^{\mathrm{a}}$ Institute of Theoretical and Experimental Physics, Moscow, Russia

${ }^{\mathrm{b}}$ Fermilab, Batavia, Illinois, U.S.A.

${ }^{\mathrm{c}}$ Tel-Aviv University, 69978 Ramat Aviv, Israel

${ }^{\mathrm{d}}$ Institute for High Energy Physics, Protvino, Russia

${ }^{\mathrm{e} C a r n e g i e-M e l l o n}$ Univ. Pittsburgh, Pennsylvania, U.S.A.

\begin{abstract}
A large-acceptance, highly segmented electromagnetic lead-glass calorimeter for Experiment E781 (SELEX) at Fermi National Acceleration Laboratory was designed and built. This detector has been used to reconstruct photons and electrons with energies ranging from a few $\mathrm{GeV}$ up to $500 \mathrm{GeV}$ in the collisions of the $600 \mathrm{GeV} \Sigma^{-}$ hyperons, $\pi$ mesons and protons with the target nucleons. The design, calibration and performance of the calorimeter are described. Energy resolution and position resolution are assessed using both calibration electron beams and $\pi^{0}$ mesons reconstructed in $600 \mathrm{GeV}$ hadron-hadron interactions. The performance of the calorimeter in selecting resonant states that involve photons is demonstrated.
\end{abstract}

Key words: calorimeter, lead-glass, radiation damage 


\section{Introduction}

During the last decade there has been significant progress in the understanding of charm hadroproduction [1]. Several results from Fermilab and CERN fixed target programs established the short lifetime of the $\Lambda_{c}^{+}$baryon, confirmed the difference in $D_{s}^{+}$production from $\Sigma$ and $\mathrm{K}$ beams and confirmed the existence of the $\Xi_{c}^{+}$baryon. From both hadronic experiments and $e^{+} e^{-}$ annihilation data emerged new evidence of $\Sigma_{c}^{++}$and $\Sigma_{c}^{0}$. However, in comparison with the meson sector, the baryons are still largely unexplored. An international collaboration of the Fermilab experiment E781 constructed a

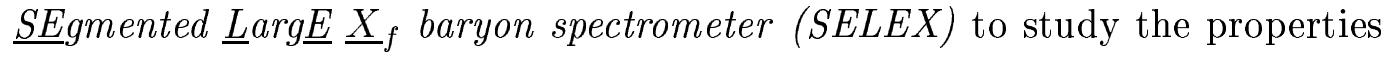
of charmed baryons produced in the hyperon beam of the Tevatron [2]. In the $S E L E X$ experiment, secondary particles are fully identified over the whole momentum range of interest for large- $x_{f}$ charmed baryons. Particular attention has been given to weak decays of their ground states, like $\Lambda_{c}^{+} \rightarrow \Lambda^{0} \pi^{+} \pi^{0}$, to radiative and strong transitions like $\Xi_{c}^{*} \rightarrow \Xi_{c}+\gamma, \pi^{0}$ and to semileptonic decays of charmed mesons. The SELEX program also includes several noncharm physics topics like Coulomb production reactions with photons in the final state.

The electromagnetic calorimeter is based on total absorption Cherenkov counters with radiators of lead-glass. It measures final state electrons and photons. Previous publications [3,4] described the prototypes of this detector. This paper focuses on the design, construction and performance of the SELEX lead-glass calorimeter, so called Photon detectors.

Following a brief review of the SELEX spectrometer as a whole in Section 2, design and construction of the Photon detectors are described in Section 3. Section 4 describes electronics for data collection, readout and triggering. Section 5 of this paper deals with the design of the Photon detectors monitoring system. Section 6 describes magnetic shielding test results. In Section 7, energy calibration of the calorimeter is described. In Section 8, the performance of the gain monitoring system during the data-taking period is presented. Finally, Section 9 addresses the general performance of the Photon detectors during physics runs at the Tevatron accelerator. The results are summarized in Section 10.

\footnotetext{
$\overline{1 \text { deceased }}$

2 E781 spokesman

3 Corresponding author: Tel + 7095129 9438; e-mail: evdokimo@itep.ru

4 Now at Northwestern University, Evanston, IL, USA
} 


\section{SELEX Setup}

The general layout of the $S E L E X$ experiment is shown in Fig. 1. The $S E$ $L E X$ spectrometer is a $60 \mathrm{~m}$ long, three stage spectrometer. The Fermilab Proton Center hyperon facility provides a variety of beams, which are brought to the SELEX spectrometer. The $600 \mathrm{GeV}$ negative beam consists of about $50 \% \Sigma^{-}$and $50 \% \pi^{-}$at $10 \mathrm{~m}$ downstream of the hyperon production target [5]. The beam spectrometer, placed in front of the charm target, is equipped with silicon microstrip detectors to track incoming particles. There are two systems - beam hardware scattering detectors (Beam HSD) and beam silicon strip detectors (BSSD) with different time selectivity (100 ns and $5 \mu$ s correspondingly), where the latter is mounted on a monument block inside a noise shielded cage (RF-cage) together with the target, followed by the vertex spectrometer formed by vertex silicon strip detectors (VSSD). Incoming particles are identified by a beam transition radiation detector (BTRD). At the end of the vertex spectrometer, vertex hardware scattering detector (Vertex HSD) is mounted to RF-cage.

The ensemble of charm targets includes carbon, silicon and copper. In addition, SELEX has an independent 3\% interaction length lead target for Primakoff reactions, located $0.5 \mathrm{~m}$ upstream of the charm targets (not shown).

The first dipole magnet is normally operated at $1.347 \mathrm{~T}$, equivalent to a $p_{t}$ kick of $0.74 \mathrm{GeV} / \mathrm{c}$. The M1 spectrometer is designed to analyze particles in the $2.5-15 \mathrm{GeV} / \mathrm{c}$ range. The tracking devices in this spectrometer consist of three proportional wire chambers M1 PWC, two drift chambers M1 DC and two stations of silicon microstrip detectors (called Large Area Silicon Detectors, LASD1 and LASD2). A lead-glass calorimeter Photon 1 provides photon detection and energy measurements.

M2 is the fast-particle spectrometer (for momentum higher than $15 \mathrm{GeV} / \mathrm{c}$ ). The $1.541 \mathrm{~T}$ M2 magnet provides a $p_{t}$ kick of $0.845 \mathrm{GeV} / \mathrm{c}$. The magnet's end gap is covered by another LASD station. Following 14 M2 PWC planes (see Fig. 1), this spectrometer also features a transition radiation detector for electron identification (ETRD) and a $10 \mathrm{~m}$ long RICH [6], followed by $10 \mathrm{~m}$ of decay region with two sets of vector drift chambers (VDC A and B) and an-

other lead-glass calorimeter Photon 2. Two trigger hodoscopes $\mathrm{H} 1$ and $\mathrm{H} 2$ are located in the M2 spectrometer as well.

The third magnet operates at $1.3 \mathrm{~T}$, equivalent to a $p_{t}$ kick of $0.72 \mathrm{GeV} / \mathrm{c}$, and is installed $42 \mathrm{~m}$ downstream of the charm target. The third spectrometer M3 enhances the acceptance for decays of long-lived hyperon states. Again there are PWCs, DCs, a lead-glass calorimeter Photon 3 and finally a hadronic calorimeter (NCAL).

The main objective of the experiment is production and spectroscopy of charmed baryons, i.e. short-lived particles of neutral or positive charge produced by a beam-target interaction. The trigger looks for a secondary vertex and at least two fast positive particles. A typical reaction is $\Lambda_{c}^{+} \rightarrow p K^{-} \pi^{+}$. 
Two hodoscopes deployed downstream of the second magnet determine the charged particle multiplicity there. Since online vertex reconstruction would be too slow for triggering, SELEX follows the idea of an impact parameter trigger: if a valid beam track and a vertex are found, the event is only accepted if they don't match. The miss distance or impact parameter is required to be larger than $20 \mu \mathrm{m}$. The SELEX trigger was designed as a four-level trigger, where the last one is a software trigger. A schematic layout of the trigger is shown in Fig. 2.

The first trigger level $T 0$ fires if a valid beam definition coincides with ready state of the apparatus. The decision of when to clear the silicon detectors is also done at the $T 0$ level. The $T 0$ signal provides the synchronization for the whole experiment.

The second trigger level $T 1$ decision is formed from BTRD and multiplicity information. Coincidence of $T 0$ with $T 1$ passes the event to the third trigger level $T 2$, which was established to include information from slower or downstream detectors in the readout.

If the event passes these three levels, all subsystems are read out into dualported memories. The event is analyzed by online filtering software running on a multi-processor SGI Challenge.

This filter process includes a partial reconstruction of the primary vertex, using the beam track and fast outgoing track reconstructed in silicon detector and PWCs after M2. If one or more high momentum tracks miss the primary vertex by at least $2.5 \sigma$, the event is written to hard disk and later to tape. Buffering the data on disk is necessary because tape drives would not be able to keep up with the peak data flow rate during the spill. Furthermore, disk buffering serves as a safety measure in the case of failing tape drives.

Along with charm physics, non-charm, or beam, physics topics are major interests within E781. There are three beam physics triggers implemented in conjunction with charm physics. The following beam triggers are generated:

- The Hadron-Electron, or $H$-e, elastic scattering trigger which looks for final states with two negatively charged particles.

- The Hyperon, or Hyp, trigger, which demands a single charged particle in the target region and three charged particles downstream

- The Exclusive, or Ex trigger, looks for final states with three to five charged particles. The photon detection system plays an important role in the low multiplicity physics probed with these triggers. This will be discussed in detail later.

\section{The Design of the Photon Detectors}

One of the main requirements for successful experiments in modern hadron spectroscopy is a possibility to detect not only charged secondaries, but neu- 
tral ones as well, i.e. photons from radiative decays of secondary hadrons: $\pi^{0} \rightarrow \gamma \gamma, \eta \rightarrow \gamma \gamma, \Sigma^{0} \rightarrow \Lambda \gamma$, etc.

The photon detection system of the $S E L E X$ spectrometer is an essential part of the experiment. It is used in different parts of the experimental program - in the study of charmed physics, electromagnetic form factors of hyperons, i.e. processes in the Coulomb field of nuclei (measurements of pion polarizability and radiative widths of hadrons, search for exotic mesons), in the search for charmed pentaquarks and cryptoexotic strange baryons with additional hidden strangeness, and so on.

\subsection{General Layout}

As was mentioned above, the $S E L E X$ spectrometer consists of three independent spectrometers called M1, M2, M3, which include Photon 1, 2 and 3 detectors. Technically, each of these sections represents a completely independent system. The E781 Photon detectors are designed to cover a large photon energy range extending to 20,50 and $500 \mathrm{GeV}$ for Photon 1, 2 and 3 respectively. Each calorimeter has a modular structure with 630, 726, 316 lead-glass counters for Photon 1, 2 and 3 respectively. Each detector features a hole for the beam that did not interact in the target box. The structure of Photon detectors is detailed in Table 1.

The beam view of Photon 1 is shown in Fig. 3. It is placed at a distance of $5.37 \mathrm{~m}$ from the center of the target box covering the polar angle range from 20 to $100 \mathrm{mrad}$. The lateral dimensions of Photon 1 are $136.9 \mathrm{~cm}$ in the horizontal direction $(X)$ and $111 \mathrm{~cm}$ in the vertical direction $(Y)$. A rectangular area of $42.5 \times 16.5 \mathrm{~cm}^{2}$, symmetric with respect to the center of the array, is left open for the fast particles that are analyzed by the downstream system.

The beam view of Photon 2 is shown in Fig. 4. It is placed at a distance of $35.67 \mathrm{~m}$ from the center of the target box subtending polar angles between 7 and $20 \mathrm{mrad}$. The lateral dimensions of Photon 2 are $230.9 \mathrm{~cm}$ in $X$ and $110.6 \mathrm{~cm}$ in $Y$. A hole of $50.7 \times 41.2 \mathrm{~cm}^{2}$ is symmetric w.r.t. the center of the array.

The beam view of Photon 3, built of the elements of the E704 detector [7], is shown in Fig. 5. It is placed at a distance of $50 \mathrm{~m}$ from the center of the target box subtending the polar angle interval between 0 and $7 \mathrm{mrad}$. The lateral dimensions of Photon 3 are $80 \mathrm{~cm}$ in $X$ and $61 \mathrm{~cm}$ in $Y$. A hole of $15.2 \times 7.6 \mathrm{~cm}^{2}$ is shifted by $13.3 \mathrm{~cm}$ to the left of the array center when looking downstream.

Each calorimeter is enclosed in metal housing. The housing protects the lead-glass detectors from outside light and magnetic fields. It also serves as a hermetic volume to flush photomultiplier bases with neutral dry nitrogen gas in order to avoid phototube instabilities caused by moisture and heating. 


\subsection{Movable Stands of the Photon Detectors}

Two different techniques were used to calibrate the Photon detector. The first technique was to expose each block to electrons of known energies. In the second approach, the energies of photons incident on the lead-glass array were determined using the kinematics of $\pi^{0}$ decays.

To perform the calibration and for beam tests, the entire assembly of each Photon detector is installed on a movable stand. These stands with movable platforms have been constructed by ITEP (Moscow) and IHEP (Protvino) and are mounted on rails in the SELEX experimental area of the Tevatron accelerator.

Electric motors (powered off $2.2 \mathrm{~kW}$ for Photon 1,2 and $3.0 \mathrm{~kW}$ for Photon 3) move the stands vertically. Each stand is equipped with limit switches for the movable platform and with string position sensors connected with the FERMILAB EPICURE [8] slow control system for computer readout. The distance between the end switches is $1000 \mathrm{~mm}$ for the Photon 1 stand, $1300 \mathrm{~mm}$ for the Photon 2 stand and $640 \mathrm{~mm}$ for the Photon 3 stand.

Power control circuitry is assembled in racks installed near each Photon detector. It contains temperature-controlled switches, relays for motor control, controls for motor direction of motion (a phase inverter), power connectors with the stands, connectors with the EPICURE computer interface rack, and light indicators of motion direction.

Tests were made to prepare the motion control system and position readout for calibration. The speed of motion is measured as $2.00 \mathrm{~mm} / \mathrm{sec}$ for Photons 1 and 2 and $4.00 \mathrm{~mm} / \mathrm{sec}$ for Photon 3 . The vertical position of the movable platform is monitored with good linearity and accuracy to better than $0.5 \mathrm{~mm}$ using string position sensors within the motion range. Details of the beam calibration procedure are given below.

\subsection{Lead-Glass Counters}

The layout of a lead-glass counter is shown in Fig. 6. Its main components are a lead-glass block and a photomultiplier.

Three types of lead-glass (see Table 2 for details) are used for Photon detectors. The 576 inner blocks of Photon 1 and 540 inner blocks of Photon 2, with dimensions of $42.5 \times 42.5 \times 340 \mathrm{~mm}^{3}$ each, are made of TF1-type lead-glass. The density of the TF1 lead-glass is $3.86 \mathrm{~g} / \mathrm{cm}^{3}$, and the radiation length of the counters is $2.8 \mathrm{~cm}$ with $5 \%$ accuracy.

The size of the inner lead-glass block is chosen for position resolution. For a photon incident on the middle of the block one wants the shower energy to spread out to neighboring counters, so that a precise measurement of shower position using the shower profile can be made. A finer sampling of the shower 
should enhance the position accuracy, while the reduced amplitude per counter leads to larger statistical fluctuations in energy. The optimal solution for shower containment is known to be a 3x3 counter, i.e., when the energy of a photon aimed at the middle of a block is fully contained in its eight nearest neighbors plus itself.

Lead-glass type F8 is used in 54 outer blocks of Photon 1 and for 186 outer blocks of Photon 2 with the sizes of $85 \times 85 \times 340 \mathrm{~mm}^{3}$. The density of the F8 lead-glass is $3.6 \mathrm{~g} / \mathrm{cm}^{3}$ and the radiation length of the counters is $3.1 \mathrm{~cm}$.

The dimensions of all 316 lead-glass blocks of Photon 3 are $38.1 \times 38.1 \times$ $450 \mathrm{~mm}^{3}$. They are made of two types (TF1 and TF101) of lead-glass with slightly different physical and chemical properties. The counters made of most radiation hard lead-glass TF101 are used for the 128 central blocks of the detector. The density of the TF101 lead-glass is $3.86 \mathrm{~g} / \mathrm{cm}^{3}$ and the radiation length of the counters is $2.8 \mathrm{~cm}$.

Degradation of the optical properties of lead-glass because of radiation damages was significant in the SELEX experiment. The choice of TF101 material for the central part of Photon 3 was motivated by its radiation hardness. We have closely monitored glass irradiation as reported below.

Cherenkov light emitted by electromagnetic showers is detected by photomultiplier tubes (PMT). PMT FEU-84-3 with a photocathode diameter of $34 \mathrm{~mm}$ are used in Photon 3 and in small counters of Photons 1 and 2. The Cherenkov light produced in the big blocks of Photons 1 and 2 is detected by PMT FEU-110 with a photocathode diameter of $68 \mathrm{~mm}$.

An ordinary magnetic shield allowed Photon 2 to work in a magnetic field up to 6-8 Gauss. As seen in Fig. 1, the distance between Photon 1 and the center of the M2 magnet is about $1 \mathrm{~m}$, and the field could reach up to 20-30 Gauss. The PMT is protected from the magnetic field by the shield made of a $\mu$-metal and soft steel. In Photons 1 and 2, the tubes FEU-84-3 and small lead-glass blocks are optically coupled via Silicon Rubber Compound RTV-615 (General Electric) lightguides with refractive index 1.405 made in the form of cookies. They are combined with plexiglass plates to provide optical contact between a big lead-glass block and FEU-110. The cookies sizes are presented in Table 3. For Photon 3, Silicon Rubber SILGARD is used. A plastic flange glued to the lead-glass block serves as a holder for the PMT to its base and as magnetic shielding. Test results of magnetic shielding efficiency confirmed that the local magnetic shielding combined with an iron box provide satisfactory magnetic screen for the Photons' PMTs. An optical fiber is attached to the front face of each lead-glass counter to deliver light pulses from the monitoring system. 


\subsection{High Voltage (HV) System}

For efficient use of HV supplies, tubes with similar gains are grouped in sets of ten. Each set is powered by one channel of an LeCroy 1440 power supply HV system. Each HV channel is independently programmable, which is particularly convenient during calibration runs, enabling separate adjustment of $\mathrm{HV}$ on groups of PMTs. Fine tuning of the HV in each PMT is done with the help of potentiometer installed on the calorimeters.

Booster power supply (BPS) modules are used to maintain PMT gain stability in Photon 3 where detector occupancy is high. These BPS modules maintain constant voltages on the last four dynodes, independent of rate. The achieved voltage stability is better than $10^{-3}$ with a beam intensity of $1 \mathrm{MHz}$.

Four modules of the BPS are housed in a single crate which moves with Photon 3. The BPS crate has a voltmeter to set the selected booster voltage in each module in sequence and to monitor the PMT current consumption during the beam run.

\section{Readout electronics}

The PMT output is directly connected via 66-m-long coaxial delay cables to the ADC analog inputs. In E781, LeCroy 1881M FASTBUS ADCs are used to read out the Photon detectors. The FASTBUS crates are housed in racks which are cooled by chilled water. The temperature of the crate is monitored by the EPICURE readout, which reads the temperature sensors installed in the rack. Inside the crate, ADC modules are controlled through FASTBUS Smart Crate Controller (FSCC) [9]. The FSCC is basically a diskless single user and multiple task computer. Each FSCC contains a Motorola 68030 microprocessor with $1 \mathrm{MB}$ RAM and an Ethernet interface.

\section{1 $A D C$}

The LeCroy Model 1881M provides 64 channels of analog to digital conversion in the FASTBUS format. It is designed for elementary particle and nuclear physics experiments and was developed to meet the fast conversion time needs of modern experiments.

The 1881M Analog to Digital Converter offers an approximately $12 \mu$ s conversion time for all 64 channels. It has a $50 \mathrm{fC}$ least count with a full 13 bits of dynamic range above the pedestal for each channel. Permissible gate widths may vary from 50 to 500 ns. Each $1881 \mathrm{M}$ has 64 channels of front panel input and a single gate input. Events are stored in an onboard 64 event cyclic 
buffer memory. A threshold memory permits the loading of a separate constant for each channel, which is used to suppress unwanted data. Both front panel control inputs are differential ECL and are terminated by a balanced $102 \Omega$ impedance matching network. The terminations may be disconnected using jumpers to allow daisy chaining of modules.

Once the control and status registers have been properly programmed, the module is in acquisition mode and ready to accept a gate pulse. The duration of the gate pulse defines the acquisition phase. The gate may be provided either via a front panel ECL input or FASTBUS TR1 and TR2. For the duration of this gate signal, each of the 64 individual inputs integrate the charge applied to them. Immediately following this acquisition phase, the data is converted to a digital representation and then placed in a multi-event buffer to await readout. If sparsification is selected, data is discarded from channels that are below their individual thresholds. The data is thus sparsified (also known as zero suppression), so that signals on the front end inputs less than the programmed threshold values are not buffered. Using this method, it is not necessary to transmit unwanted data over FASTBUS.

A fast clear may be applied to the module at any time from 100ns after the end of the gate until the end of the Fast Clear Window (FCW). If a fast clear is applied during this period, the event currently being converted will be discarded. There is no restriction within the $1881 \mathrm{M}$ of the maximum FCW that may be applied externally. Clears should not be applied outside of the fast clear window.

The data of the $1881 \mathrm{M}$ is stored as a 32-bit word, each of which contains charge data, channel number, the geographic address and a word parity bit.

\subsection{Local Trigger Logic for the ADC}

The function of the local trigger logic for the ADCs is to take the trigger signals from the global trigger logic and fan them out into the proper shape with proper timing to the ADC modules. It should satisfy the following requirements:

- The gates must be timed to include all of the signal but not be too wide that they include noise.

- A clear signal must be generated once the event is rejected. The next gate can come in no less than 800 ns later and this time is controlled by the global trigger logic.

- As long as the signal is being processed, a BUSY signal must be generated to prevent further global trigger signals from occurring. This status will be maintained until the LeCroy $1881 \mathrm{M}$ enters the data into its buffer and is ready to process the next event. The length of this time period is fixed at $15 \mu$ s at ADC local trigger logic. 
- The BUSY signal is also generated when the ADC has too many unread events in its buffer. In the design, the latter number is limited to 32 , half of the number of events that the ADC can handle.

A simple logic was designed to attain the above goals. As shown in Fig. 7, the trigger units consist of a gate generator (LeCroy 222), discriminators (LeCroy 623B), linear and logical fan-out modules (LeCroy 428F and 429A) and NIMECL convertors (LeCroy 4616).

The pulse $T 1$ arrives to the input of the discriminator and is distributed to the LeCroy $428 \mathrm{~F}$ and $429 \mathrm{~A}$ modules. The outputs from these fan-out modules are then sent to LeCroy 4616 to be converted into ECL signals, required by the ADC modules.

After $T 1$ comes and gates to ADC are generated, conversion of the signals is started in LeCroy $1881 \mathrm{M}$. The gate is generated some $30 \mathrm{~ns}$ after arrival of $T 1$ at the discriminator. To obtain correct timing, the trigger pulse is delayed before reaching the ADC modules. (This is indicated by the vertical dashed line in Fig. 7.) Because this delay should be the same for those ADCs which read out data from the same Photon, the delay time was adjusted detector by detector.

The fan-out modules have multiple ORed inputs. Therefore, in addition to $T 1$ input, the other sources were also connected. These include the gates for probing the pedestal, for LED testing and for laser testing. These inputs also were properly delayed before reaching this point.

The next trigger signal is either $T 2$ or $\bar{T} 2$, depending on whether the event is accepted or rejected. In the accepted event case, the $T 2$ is connected to the inputs of two LeCroy 222 gate generators. The first generator is set up for a $14 \mu$ s delay. The delayed output is used as the strobe to the FSCC, which tells the FSCC to start reading data from the ADC. The NIM output of the second LeCroy 222 is taken as the BUSY signal and sent back to the global trigger system to inhibit the next trigger while the conversion is underway. This BUSY signal lasts for approximately $15 \mu \mathrm{s}$. It is also ORed with two outputs called THO (Trigger Hold Output) from the FSCC. THO is set up in such a way that once there are more than 32 events accumulated in the ADC buffer it becomes TRUE. Therefore, when there are 32 unread events in the ADC buffer, the BUSY will take effect until the next event is read out from $\mathrm{ADC}$. The $1 \mu \mathrm{s}$ difference between these two gate generators eliminates the gap between the ADC conversion BUSY and the FSCC readout BUSY.

In the case of the event being rejected, $\bar{T} 2$ will generate clear signals for all ADCs to clear the data. After clearing, the ADCs are ready to accept the next event.

The timings for these two situations are illustrated in Fig. 8. In this picture $T 12$ is determined by global trigger logic, defining time difference between $T 1$ and $T 2 . T d$ is delay adjusted detector by detector to obtain the correct timing. 


\subsection{Photon 3 Trigger}

As mentioned above, the total energy deposit in Photon 3 was included in the $T 2$ trigger logic. The scheme of the trigger is presented in Fig. 9. For the tubes in each column a sensitive divider sends $5 \%$ of the current to a linear shaper. The column sums are added in a second unit. Finally, a discriminator generates the $T 2$ trigger signal if the energy exceeds threshold.

\section{Overview of the Monitoring System}

Changes in the temperature of the PMT and the electronics, as well as glass transparency degradation due to radiation damage, can cause long and short term variations of the amplitudes of signals read out from the lead-glass counters. Therefore, calibration constants of each lead-glass counter must be monitored throughout the long periods between electron beam calibration. The monitoring system is responsible for the following tasks:

- Monitoring of the readout of all channels by sending short light pulses during and between beam bursts.

- Linearization of each readout channel. The linearity of the response is checked over the full dynamic range. For channels with non-linear response, appropriate corrections are introduced.

The scheme of the monitoring system is shown in Fig. 10. It includes two independent systems based on Light Emitting Diodes (LEDs) and on a highpowered ultraviolet (UV) laser.

\subsection{LED Monitoring System}

The LED monitoring system delivers short pulses via optical fibers to each counter. The main elements of this monitoring system are:

- the light distribution system,

- the system of LED light pulse sources (red, orange),

- the system of PIN photodiodes with high stability.

The light distribution system supplies light pulses with fixed amplitudes into each of 1680 readout channels. As mentioned above, the front panel covering the lead-glass array has a $1 \mathrm{~mm}$ hole for inserting a single optical fiber, that is fixed by a rubber washer and a nut. The front plane is protected from external light by a removable cover. The light distribution system is installed in the 
space between the front plane and the cover.

Each calorimeter has a light distribution box (LB) which mixes an input light pulse from an optical source into quasi-uniform light pulses on the optical fiber network that goes to each lead-glass block. The light box consists of a cylindrical plastic box, with length of $\sim 10 \mathrm{~cm}$ and diameter of $\sim 4 \mathrm{~cm}$, and of a light source.

The light source is based on red LEDs ${ }^{5}$ for Photons 1 and 2 and on red and orange LEDs for Photon 3. Each LED is fired by a short (100 ns) signal provided by a pulser.

The fiber bundles for Photons 1 and 2 consist of glass fibers with diameter of $\sim 2 \mathrm{~mm}$ and length of $\sim 200 \mathrm{~cm}$. The fiber bundle for Photon 3 consists of plastic fibers that are glued together forming a rigid structure with a diameter of $\sim 3 \mathrm{~cm}$. The fiber ends are cut and polished to provide good light transmission. Each fiber has a diameter of $\sim 1 \mathrm{~mm}$ and a variable length of $\sim 20$ to $80 \mathrm{~cm}$, adjusted so as to reach all Photon 3 channels.

Because the LED output intensity varies considerably, pulse height normalization is done using photodiodes. Two photodiode/preamp (PD) circuits housed in RF-shielded boxes are optically coupled to the light distribution box via fibers. The photodiode assembly consists of two identical channels. Two PIN photodiodes couple to a fast preamplifier, specially designed for $S E$ $L E X$ by the Tel-Aviv University group. The photodiode Hamamatsu S1223-03 has been chosen, as it has a sufficiently large bandwidth of $20 \mathrm{MHz}$, a large active area of $3.6 \times 3.6 \mathrm{~mm}^{2}$, a large spectral range of $320-1100 \mathrm{~nm}$ and a low temperature coefficient of $\sim 0.1 \% /{ }^{\circ} \mathrm{C}{ }^{6}$. The incident light pulse is injected via optical fibers and focused by small lenses on the active surface

of the diodes. Their responses to incident light are linear over the range of equivalent light-energy of each calorimeter, and stable over long periods of continuous operation. The monitoring system provides normalization of the photodiode signals with $<1 \%$ stability.

\subsection{Laser Monitoring System}

As mentioned above, the Photon detectors are positioned at large distances from the target box and have very different dynamic ranges. They operate in the energy range 1-500 GeV. In order to overcome transmission losses while still delivering a large dynamic range of energy-equivalent light intensity requires a laser light source. The laser system, based on a Laser Photonics LN300C, is used to monitor all three Photon detectors simultaneously, to perform phototube readout linearity and timing tests and to simulate the SELEX

$\overline{5}$ red LED, type AND190GRP, $10 \mathrm{~mm}$ package, I(MCD@20mA)=13000, view angle $4^{\circ}$, manufactured by Hewlett Packard

${ }^{6}$ Hamamatsu photodiodes, Cat. KPD0001E02, Feb. 94 
trigger for Photon detectors in the absence of beam. Its main elements are:

- a high-power $\mathrm{UV} \mathrm{N}_{2}$ laser coupled to a dye laser module,

- a remotely controlled wheel containing several neutral density filters to attenuate the laser beam,

- the first light distribution stage located near the laser, which splits the beam into fibers running out to all Photon detectors.

The primary laser light distribution system (see Fig. 10(b)) consists of a laser and a simple optical system to control the amplitude/timing of the light pulse and then deliver it to the light distribution system via a long optical fiber.

The $\mathrm{N}_{2}$ laser with an energy of $280 \mu \mathrm{J} /$ pulse emits short $4 \mathrm{~ns}$ pulses with a wavelength of $337 \mathrm{~nm}$ at a rate of 1-30 Hz. It is used for pumping a dye laser. A blue dye with peak emission at $425 \mathrm{~nm}$ and a conversion efficiency of $20 \%$ is used. The colored light beam enters an aluminium box, which contains the first PIN photodiode used for timing (PD-T), a computer controlled electromagnetic shutter, a set of 12 calibrated neutral density filters mounted on a computer controlled wheel, a second PIN photodiode measuring the pulse amplitude (PD-A) and, finally, the injector of the light pulse into an optical fiber with the diameter of $1 \mathrm{~mm}$ and length of $\sim 80 \mathrm{~m}$.

The light splitter distributes the laser light pulse to the calorimeters in proportion to their dynamic ranges and number of lead-glass blocks.

As Photon 1-3 detectors have dynamic ranges of 20,50, $500 \mathrm{GeV}$ and consist of 630,726 and 316 lead-glass counters, respectively, the light pulse amplitude is split in a proportion of $7 / 20 / 100$.

\subsection{Monitoring Procedure}

The accelerator supplies beam particles for $20 \mathrm{~s}$ out of each $60 \mathrm{~s}$. The time between bursts is used for monitoring. At interspill time gates are provided via CAMAC by Fermilab 040 Pulse train units with three independent outputs each, located in the CAMAC crate (Fig. 11). Output 1 provides the gate signal for the $\mathrm{ADC}$ pedestal measurement with the same electronics as in $T 1$, while Output 2 is split into two signals: the first is used to trigger the LED signal and the second to generate the gate measuring the detector response.

Every detector has its own LED system, which produces light pulses and distributes them over the detector. The second signal, after a certain delay, is delivered to the LeCroy 623 discriminator and then to the $T 1$ gate system. Output 3 is used for laser measurement. It sends the trigger signal to the laser and at the same time produces the gate to measure the pedestals. The laser trigger signal is sent over about $100 \mathrm{~m}$ cable to the laser, where it is converted to a TTL $5 \mu$ s-wide signal. On this trigger, the laser fires and the light pulse is transported back to the experimental hall via an optical fiber and delivered to 
Photons 1,2 , and 3. The laser light pulse is also detected by two photodiodes (PD-T and PD-A) as described in the previous section. The PD-T fast timing signal is sent to the experimental hall synchronously with the light pulse and detected by the discriminator to be fed to the rest of the $T 1$ system and to produce the $\mathrm{ADC}$ gate.

The ADC calibration monitoring program, mon, is used to record basic parameters of the detectors. The program runs independently on the FSCC computer in the ADC FASTBUS crate under the control of the master crate resident program. Master spawns mon, awakes it during interspill time and sends it to sleep during the spill. For every ADC channel connected to the detector, mon computes the following values:

- the average ADC response,

- the RMS of the external light signal, either LED or laser,

- the ADC response without any light signal (pedestal).

The program estimates the quality of these values and compares them with those obtained earlier, which are considered as a stable reference. These earlier data are stored in a work database. Mon also produces an output file, histoscope files used to evaluate the detectors and files containing the ADC thresholds. These thresholds are set slightly above the pedestal's mean values, to be used to suppress noisy channels in the ADC sparse mode used for data taking.

\section{Test of the Photon Detectors}

As mentioned above, in the SELEX experiment the PMTs experienced significant stray magnetic fields. The magnet nearest to Photon $1, \mathrm{M} 2$, generates a magnetic field $H \leq 13 \mathrm{kG}$ (absolute value) in the magnetic gap. The LED monitoring system is used to study the field effect. The amplitudes of LED signals were obtained for the following four cases:

1. $A_{\text {initial }}$ for magnetic field $\mathrm{H}=0$, before switching the magnet on;

2. $A_{+}$for $\mathrm{H}=+13 \mathrm{kGs}$, positive current $\mathrm{I}=+2500 \mathrm{~A}$;

3. $A_{-}$for $\mathrm{H}=-13 \mathrm{kGs}$, negative current $\mathrm{I}=-2500 \mathrm{~A}$;

4. $A_{\text {final }}$ for $\mathrm{H}=0$, after switching the magnet off.

We measured the following amplitude changes:

$$
\begin{aligned}
& \Delta_{+i}=\left(A_{+}-A_{\text {initial }}\right) / A_{\text {initial }}=(-22 \pm 4) * 10^{-4} \\
& \Delta_{-i}=\left(A_{-}-A_{\text {initial }}\right) / A_{\text {initial }}=(-0.4 \pm 3.5) * 10^{-4} \\
& \Delta_{+-}=\left(A_{+}-A_{-}\right) / A_{-}=(-19 \pm 4) * 10^{-4} \\
& \Delta_{+f}=\left(A_{+}-A_{\text {final }}\right) / A_{\text {final }}=(24 \pm 3) * 10^{-4}
\end{aligned}
$$




$$
\begin{aligned}
& \Delta_{-f}=\left(A_{-}-A_{\text {final }}\right) / A_{\text {final }}=(3 \pm 4) * 10^{-4} \\
& \Delta_{f i}=\left(A_{\text {final }}-A_{\text {initial }}\right) / A_{\text {initial }}=(5 \pm 2) * 10^{-4}
\end{aligned}
$$

All differences in amplitudes are well below 1\%. These results confirm that the local magnetic shielding combined with the iron box of the detector provide satisfactory magnetic screening for the Photon PMTs.

\subsection{Space Resolution Studies}

Space resolution was estimated using electrons from the calibration data set. Detector space resolution is influenced by the transverse dimensions of the electromagnetic shower as well as by the size of the lead-glass counter. To measure the shower profile, a ratio of the amplitudes in two neighboring blocks was measured as a function of the coordinate of the entry point of the electron beam. The transverse size of the shower is determined by the Moliere radius $R_{M}$,

$$
R_{M}=X_{0} E_{S} / E_{C}
$$

where the scale energy $E_{S} \sim 21 \mathrm{MeV}, E_{C}$ for the TF1 lead-glass block is $15 \mathrm{MeV}$ and $X_{0}$ is $2.5 \mathrm{~cm}$. The observed sizes of showers are consistent with a Moliere radius value of $R_{M}=3.5 \mathrm{~cm}$ for the lead-glass.

The data of calibration runs were used to estimate the value of space resolution of the detector as a function of beam position. The position resolution was found to vary in the range $2.0-5.5 \mathrm{~mm}$, improving from the center towards the edge of the central block.

\subsection{Depth Correction Studies}

If the photon angle of incidence is not $90^{\circ}$, the transverse position of the photon impact point into the calorimeter and of the shower maximum are not the same. The corresponding correction can be derived experimentally by studying the showers from electrons hitting the lead-glass at different angles.

Electrons were taken from charm trigger runs and from runs with a special trigger (Section 2). SELEX track reconstruction determines the trajectory, its entry point $r_{a c t}$ and angle $\theta$ in the lead-glass block. The center of gravity of the electron shower $\left(r_{\text {clus }}\right)$ is calculated. The depth correction $\left(r_{c l u s}-r_{a c t}\right)$ defines the shower depth:

$$
D=\left(r_{c l u s}-r_{a c t}\right) / \sin \theta \text {. }
$$


It was found that the best average value of the shower $\operatorname{depth} D$ over all energies for Photon detectors is about $16 \pm 3.5 \mathrm{~cm}$. For Photon 3 this correction is unnecessary, it accepts only photons with angles $<8 \mathrm{mrad}$. 


\section{Calibration of the Photon Detectors}

\subsection{Event Reconstruction}

The algorithms used to reconstruct particles in lead-glass calorimeters are described in [10] and [11]. The Photon detectors reconstruction program is integrated into the SELEX Offline Analysis Program (SOAP). The Photon detectors database contains information about the geometry, ADC-block mapping, PMT gains, pedestals and other data needed for reconstruction. Reconstruction starts by finding the hit counters and their energy depositions. If the energy deposited in the counter exceeds a certain limit, the corresponding channel is used further to form first stage clusters. In the next step, the program searches for coordinates of local maxima in each cluster. Next, the impact point of the particle that hit the calorimeter is reconstructed.

When several photons hit the calorimeter close to each other, the problem of reconstruction becomes more complicated due to overlapping of clusters. One of the reconstruction methods is to separate such clusters and fit twodimensional functions with the set of detected amplitudes, varying the impact coordinates and photon energies. Unfortunately, this algorithm is very CPUintensive, hence an alternative method is used.

After finding the maxima in the first stage clustering, the comparison of each shower shape with the ideal one is made in terms of $\chi^{2}$. The database contains shower profiles for several fixed energies for three types of lead-glass blocks according to the type of the glass and the block size. The aim of this database is to describe the shape of the shower in terms of the cluster center of gravity coordinates. If there is a cluster with a center of gravity coordinate $\left(X_{C}, Y_{C}\right)$ and a cell (counter) with the coordinates $(\mathrm{X}, \mathrm{Y})$, then the function phot_cell $\left(X-X_{C}, Y-Y_{C}\right)$ returns the energy in the cell for the case when the shower is electromagnetic and ideal (no fluctuations). The program then compares this value with the measured one and calculates $\chi^{2}$. If this $\chi^{2}$ is satisfactory $\left(\chi^{2} \leq \chi_{\max }^{2}\right)$, the cluster is accepted as a photon, otherwise separation using an iterative procedure is performed, introducing a second photon peak, based on the positions of local peaks. The cluster matches neutral hadrons if $\chi^{2}$ remains high after all iterations. For particle identification, track information from the SELEX spectrometer is used to estimate the impact point of all charged tracks. For each cluster, the difference $x_{r}$ between the nearest impact point and the cluster center is calculated. If $x_{r}$ exceeds the limit $h_{r}$ (maximum hadronic linkage radius) the cluster is considered as a photon. Usually $h_{r}=5 \mathrm{~cm}$, but the value of $h_{r}$ may depend on physics goals. A cluster

with $x_{r} \leq h_{r}$ is classified as electron, muon or charged hadron depending on $\chi^{2}$ values of energy, reconstructed momentum and cluster dimensions. For clusters identified as photons an additional correction is applied to the $(\mathrm{X}, \mathrm{Y})$ coordinates based on the Lednev formula [10], which helps to remove bias and 
improve coordinate resolution.

\subsection{Detector Calibration}

The calorimeters were calibrated using dedicated electron beam runs several times during the data taking. Generally, each calibration represents a series of exposures to electrons of known energies in order to obtain calibration constants for all channels of the Photon detectors. During calibration, moving platforms shifted the calorimeter in the vertical direction in order to place each counter in the beam. Horizontal deflection was provided by the experiment magnets. However the calorimeter response to photons and electrons is slightly different [12]. To obtain more accurate calibration constants, $\pi^{0}$ data from real events is used. The next step includes selecting two-photon events and performing calibration using two-photon constrained fitting.

\subsection{Event Selection for $\pi^{0}$ Calibration}

To perform a $\pi^{0}$ calibration, the Exclusive trigger data have been analyzed. Two-photon events with each photon having an energy of at least $5 \mathrm{GeV}$ and with total energy above $15 \mathrm{GeV}$ are selected. The dimension of the clusters produced by photons has been chosen to be more than two counters in order to reduce the background. A photon candidate that has same y-coordinate as another reconstructed particle in the same Photon detector within $4 \mathrm{~mm}$ is rejected; it has too large a probability of being affected by bremsstrahlung, scattering or gamma conversion. If less than two photons in Photon 2 and Photon 3 survive after applying the cuts mentioned above, an additional search for photon is performed in Photon 1 with a lower photon energy threshold of $0.5 \mathrm{GeV}$. To reduce the size of output data, events with invariant mass of two photons less then $50 \mathrm{MeV}$ are rejected. Events with total multiplicity greater than 15 are rejected in order to reduce combinatorial background.

Initial calibration constants are computed from the electron calibration corrected by LED monitoring:

$$
G=G_{0} \times \frac{A^{L E D}}{A_{0}^{L E D}},
$$

where $G$ and $G_{0}$ are the gains for the current and calibration runs respectively and $A^{L E D}$ and $A_{0}^{L E D}$ are the amplitudes of the corresponding LED signals.

Next, from the set of events selected for $\pi^{0}$ calibration, events in which a single counter has more than half of the cluster energy are selected. The $\gamma-\gamma$ 
mass distribution is fitted by a polynomial with a Gaussian function. The gain of counter $i$ is corrected iteratively :

$$
G(k+1)_{i}=G(k)_{i} * \sqrt{M\left(\pi^{0}\right)_{P D G} / M\left(\pi^{0}\right)_{f i t}} .
$$

where $k$ is the number of iterations performed, $M\left(\pi^{0}\right)_{P D G}$ is the $\pi^{0}$ mass from the PDG [13] and $M\left(\pi^{0}\right)_{\text {fit }}$ is the $\pi^{0}$ mass calculated for $\pi^{0}$ candidates in any given event. The iteration sequence converges in about five iterations. Photon 2 and Photon 3 were calibrated first, then calibration for Photon 1 was done. Finally, the same procedure was performed on standard runs with the Charm trigger. New tables with gains are created for each group of about 200 runs.

\section{$7.4 \pi^{0}$ Calibration Results}

The parameters of the di-gamma invariant mass spectrum are obtained for a new set of calibration coefficients using two-photon constrained fitting. The only cut used here is the cut on the photon energy and the mass spectrum is fitted with a simple Gaussian distribution. The results of fitting the $\pi^{0}$ mass for events obtained with the Charm trigger are presented in Fig. 12 and Table 4. The first column in the table shows particular combinations of Photon detectors forming $\pi^{0}$ candidates. Here we consider only isolated photons with $E_{\gamma}>2 \mathrm{GeV}$ and not near the border or hole of detectors. The typical systematic $\pi^{0}$ mass shift is less than $0.5 \mathrm{MeV} / \mathrm{c}^{2}$ except in Photon 1 where

the shift is $1.2 \mathrm{MeV} / \mathrm{c}^{2}$. As expected Photon 2 yields the best spatial and energy resolutions for photons. 


\section{Monitoring the Photon Detectors During Data Taking}

The long-term stability of each calorimeter was checked by monitoring the values of the ADC pedestals and the gain values. It was found that they were stable to within a few percent during the entire time of operation. Fig. 13 shows the values of pedestals observed over a period of several months' running for three typical modules. When the pedestal values changed significantly, the database was updated. The largest variations are usually related to runs recorded shortly after long shutdowns; some time is needed for electronics to reach normal temperature conditions.

\subsection{Monitoring the Radiation Damage}

Several studies of the radiative damage were performed for different types of lead-glass Cherenkov counters in photon spectrometers, using different beams of high energy particles. Lead-glass radiation damage in SELEX Photon detectors is monitored with two light sources: the light emitting diode LED which provides red light and the dye laser (LSR) tuned to green light. It was found that in our range of exposed dose, red light from the LED passes through the glass without considerable attenuation and even after one year of glass irradiation the attenuation does not change significantly. So the relative variation of the PMT signal indicates instabilities of the PMT or of other electronics.

Green laser light passing through the glass has very little attenuation if the lead-glass block is not damaged. After substantial irradiation (a few hundred rad for standard lead-glass and about $10000 \mathrm{rad}$ for radiation hard glass), the block transparency drops for the green light. Comparison of the signals from the LED and LSR sources allows one to determine the PMT instability and glass radiation damage.

Two monitoring measurements were performed: the first one during the first Photon detectors calibration runs (December 1996) and secondly when the data taking was finished (September 1997). The distributions of the uncorrected ratios of laser signals with photodiode normalization are given by

$$
R_{\text {uncorr }}=\frac{A_{\text {laser }}^{\prime}}{A_{\text {laser }}}
$$

where $A_{\text {laser }}$ and $A_{\text {laser }}^{\prime}$ are the values of the PMT laser signals at the beginning (December 1996) and end (September 1997) measuremnets respectively, normalized by the corresponding amplitudes of the photodiode (PD-A) signals. These distributions, presented as dashed histograms in Fig. 14, show broad variations of counter signals. To estimate the effect of radiation damage, an 
additional correction using corresponding LED signals is made:

$$
R_{\text {corr }}=R_{\text {uncorr }} \times K_{\text {corr }}
$$

where

$$
K_{c o r r}=\frac{A_{L E D}}{A_{L E D}^{\prime}}
$$

Here $A_{L E D}$ and $A_{L E D}^{\prime}$ are the values of the PMT LED signals at the beginning (December 1996) and end (September 1997) measurements respectively, normalized by the corresponding amplitudes of the photodiode (PD-A) signals. It is expected that the blocks damaged by radiation have values of $R_{\text {corr }}$ that are below unity due to transparency losses as a result of radiation damage. Distributions of $R_{\text {corr }}$ also illustrated in Fig. 14 (solid histograms). Distributions of the correction coefficients $K_{\text {corr }}$ are shown in Fig. 15 (a), (b) and (c) for Photon 1, 2 and 3 respectively. Other instabilities should have a symmetric distribution, as was seen for Photon 2 big block counters. At the same time, for partially irradiated small counters the distributions are distorted since damaged counters have low values of $R_{\text {corr }}$. Positions of these counters in Photons 2 are presented in Fig. 16. The correlation between $R_{\text {corr }}$ and radiation flux from the beam is clearly seen. Positions of partially irradiated counters in Photons 3 are shown in Fig. 17. The border between radiation hard and ordinary glass is clearly seen.

Lead-glass TF101 irradiated by a dose of $2 \times 10^{4}$ rad loses about $1 \%$ of the incident light within one radiation length [14]. For TF1 lead-glass, a similar light loss is caused by an absorbed dose of $2.5 \times 10^{2} \mathrm{rad}$ [15].

In Photon 2, only two counters have lost the transparency by a factor of two or more. Applying the approximation from [15] to our glass sizes, the absorbed dose in these counters is estimated to be about $500 \mathrm{rad}$. For the counters with a transparency loss of $10 \%$ or less $\left(R_{\text {corr }}=0.8 \div 0.9\right)$ the absorbed dose is about $120 \mathrm{rad}$. Several dozen counters have smaller but measurable damage.

In Photon 3, there is no visible damage of inner radiation-hard glass blocks except for three blocks located near the beam hole. The estimated absorbed dose in these counters is $4 \times 10^{3}$ rad. Radiation damage of TF1-000 glass blocks placed along the border with inner radiation-hard blocks is evident, but it is partially masked by other instabilities.

There are several counters with a small $R_{\text {corr }}$ in Photon 1 , but the distribution is too broad to see details of their radiation damage. 


\subsection{Monitoring the Lead-Glass Transparency During Measurements}

Variations of $R_{\text {corr }}$ during the measurements have been studied for several channels of each of the Photon detectors in order to understand the dependence of lead-glass transparency on beam intensity. The results are presented in Fig. 18 for several groups of runs where the data were available. $R_{\text {corr }}$ is shown for a typical counter of Photon 1 (undamaged) and for two damaged counters of Photon 2 and Photon 3. Photon 1 counter has practically no monotonic variations in time apart from fluctuations to be considered below. For the counters damaged by radiation, a linear decrease of the signal with run number is observed. There are several special points in Fig. 18. There is a jump in the region of the runs 9837-9844 where a power outage took place. At that moment beam intensity decreased by a factor of ten. Correspondingly, $R_{\text {corr } r}$ varies slowly after this point. 


\section{Performance of the Photon Detectors}

\subsection{Experimental Data}

The main aim of the $S E L E X$ experiment is to make a systematic survey of charm baryon production and decay mechanisms. Such a dataset is needed to understand if perturbative QCD can account for charm production under different circumstances and to establish which mechanisms dominate in the decay processes. Having completed particle identification for hadrons, electrons and photons, one can normalize the branching ratios to the semileptonic rates. This aids the theoretical analysis of the hadronic modes. The ability to measure $\pi^{0}$ and $\eta^{0}$ states is also important in analyzing these ratios for evidence of resonant substructure. This substructure is clearly a dominant feature of meson decay.

Also, as has been mentioned earlier, three of the expected ground state charm baryon multiplet properties have been measured with low precision: $\Sigma_{c}^{+}, \Xi_{c}^{+}$and $\Omega_{c}^{0}$. SELEX acquired a large dataset containing various decay modes of all singly charmed baryons. In excited charm baryons the Q-value of the decay is small. Therefore the deexcitation particle is slow, emitted near the baryon line of flight. For photon decays, this means that one must find a low energy photon near $0^{\circ}$ and separate it from the bulk of the primary interaction $\pi^{0}$ decays.

Due to the $50 \mathrm{~m}$ length of the $S E L E X$ set up (which results in good small angle resolution), the long run period, excellent tracking and the high beam energy, Primakoff physics measurements with very small momentum transfer are also feasible. Among these physics interests, we mention radiative widths of hadron resonant states, hadron radius and pion polarizability.

All goals mentioned above require a high efficiency of photon and $\pi^{0}$ reconstruction. To demonstrate detector sensitivity and resolution, signals from several well known particles decaying into photons are studied. The simplest test of the calibration of the Photon detectors over a wide energy range is provided by combining mass plots for events from real data. The Exclusive trigger had lower multiplicities (three charged tracks from the vertex and about 2.4 photons with energy above $2 \mathrm{GeV}$ on average) and allowed us to obtain clear signals of many hadronic states.

$\pi^{0}$ and $\eta$ production were identified via their decay modes involving two photons in the final state. For this purpose we selected events in the Exclusive trigger in the $\pi^{-}$beam with two photon clusters, each having an energy in excess of $2 \mathrm{GeV}$ and energy of two-photon system $E_{\gamma \gamma}>10 \mathrm{GeV}$. Fig. 19 shows the invariant mass distribution of the two photons with sharp $\pi^{0}$ and $\eta$ peaks.

The $\eta$ and $\omega$ mesons were separated in their decay to $\pi^{+} \pi^{-} \pi^{0}$. For this purposes we selected events with the Exclusive trigger in the $\Sigma^{-}$beam. These 
events contained two photon clusters, each having an energy in excess of $2 \mathrm{GeV}$ and satisfied $\pi^{0}$ identification criteria (within a mass window of $\pm 25 \mathrm{MeV}$ of the PDG value [13]), together with the requirement that the $\pi^{-}$is not a leading particle. Fig. 20 shows the invariant mass distribution of $\pi^{+} \pi^{-} \pi^{0}$ for events with $E_{\pi 0}>10 \mathrm{GeV}$.

The $\eta^{\prime}$ and $f$ mesons were selected in the decay $\pi^{+} \pi^{-} \eta$. For this purpose we selected events with the Exclusive trigger in the $\pi^{-}$and $\Sigma^{-}$beam. These events contained two photon clusters, each having an energy in excess of $2 \mathrm{GeV}$ and satisfied $\eta$ identification criteria (within a mass window of $\pm 50 \mathrm{MeV}$ of the PDG value), together with the requirement that the $\pi^{-}$is not a leading particle. Fig. 21 shows the effective mass distribution of $\pi^{+} \pi^{-} \eta$ for events with $E_{\eta}>10 \mathrm{GeV}$.

In order to separate a signal from the decay $K^{0} \rightarrow 2 \pi^{0}$ we selected events with the Exclusive trigger in the $\Sigma^{-}$beam. These events contained photon clusters having an energy in excess of $4 \mathrm{GeV}$ and a number of pairs which satisfied the $\pi^{0}$ identification criteria (within a mass window $\pm 20 \mathrm{MeV}$ of the PDG value) and did not exceed four. Fig. 22 shows the effective $2 \pi^{0}$ mass distribution for events with $E_{\pi^{0}}>30 \mathrm{GeV}$. The mass resolution is slightly worse due to uncertanties in the true kaon decay z-position.

The $\eta$ meson was also separated in the decay $\eta \rightarrow 3 \pi^{0}$. In this case we required that Photon detectors recorded six photon clusters having an energy in excess of $5 \mathrm{GeV}$ and that these clusters formed three $\pi^{0}$ candidates (within a mass window $\pm 20 \mathrm{MeV}$ of the PDG value). Fig. 23 shows the effective $3 \pi^{0}$ mass distribution for events with $E_{\pi^{0}}>20 \mathrm{GeV}$.

Fig. 24 shows the effective mass distribution for the $\pi^{+} \pi^{-} \gamma$ system. For this plot we selected events with the Exclusive trigger in the $\Sigma^{-}$beam with the following cuts: the $\pi^{-}$was not a leading particle, $E_{\gamma}>2 \mathrm{GeV}$, there was at least one photon with $E_{\gamma}>50 \mathrm{GeV}$, the $\pi^{0}$ wasn't formed with any other photons, and there was only one photon with $E_{\gamma}>4 \mathrm{GeV}$ in the event. Four peaks were clearly observed (see figure for their description).

In the Charm trigger where event multiplicities are much higher (10.5 charged tracks in the vertex region and six photons on average with energy above $2 \mathrm{GeV}$ ), the photon identification is slightly more difficult harder but still possible. In the Charm trigger we selected events with photon clusters having an energy in excess of $2 \mathrm{GeV}$. Fig. 25 shows the invariant mass distribution for the energy of the two-photon system with the number of photons $N_{\gamma}<10$ and $E_{\gamma \gamma}>10 \mathrm{GeV}$. A $\pi^{0}$ signal is clearly seen in this distribution, confirming our ability to do charm physics with neutral components in final states. Of course, the cut on photon multiplicity strongly suppresses background. For the same data Fig. 26 shows the invariant mass distribution for the two-photon system with energy $E_{\gamma \gamma}>10 \mathrm{GeV}$ and the number of clusters $N_{\gamma}<3$.

In the Charm trigger sample we can reconstruct hyperons even without a cut on number of clusters. Fig. 27 shows the effective mass distribution for the $\Lambda \gamma$ system with a cut on the photon energy $E_{\gamma}>6 \mathrm{GeV}$. No cut on the 
number of photons is applied. Photon 1 is not included. The $\Lambda$ is reconstructed in the process $\Lambda \rightarrow p \pi^{-}$and constrained to the PDG mass value 1115.68 $\mathrm{MeV}$ within a $1116 \pm 3 \mathrm{MeV}$ mass window.

$D^{* 0}$ mesons decaying into identified $D^{0}+\pi^{0}$ and $D^{0}+\gamma$ are studied. The $D^{0}$ candidates were reconstructed in four modes corresponding to the processes $D^{0} \rightarrow K^{-} \pi^{+}, \overline{D^{0}} \rightarrow K^{+} \pi^{-}, D^{0} \rightarrow K 3 \pi, \overline{D^{0}} \rightarrow K 3 \pi$. Fig. 28 shows the mass difference distribution for $\Delta M_{\gamma}=M\left(D^{0} \gamma\right)-M\left(D^{0}\right)$ with the cut on photon energy $E_{\gamma}>6 \mathrm{GeV} . \Delta M_{\gamma}=0.1412 \pm 0.0009$ Photon pairs with an invariant mass within $\pm 15 \mathrm{MeV} / c^{2}$ of the nominal $\pi^{0}$ mass are accepted and kinematically constrained to its value. The results are shown in Fig. 29: $\Delta M_{\gamma}=0.1420 \pm 0.0012$. The position of the observed peak is in agreement with the known mass difference between $D^{* 0}$ and $D^{0}$.

\section{Summary}

The design and construction of three Photon detectors were described. These calorimeters were built as part of the Fermilab SELEX experiment E-781, a study of charmed baryons in a hyperon beam. The performance and stability of the calorimeter response were continuously measured during the year of data taking and the data are in good agreement with the design values and expectations. Good energy and small angle resolutions enable us to perform a variety of precise measurements in both charm and non-charm physics areas.

\section{Acknowledgments}

The authors are grateful to the staff of IHEP, ITEP, Fermi National Accelerator Laboratory, Carnegie Mellon University and Tel Aviv University for technical support. Special thanks are due to Linda Stutte for her contribution in establishing ITEP-IHEP participation in the SELEX experiment and Amber Jenkins (Imperial College, London) for invaluable editorial comments. This project was supported in part by the Israel Science Foundation founded by the Israel Academy of Sciences and Humanities, the International Science Foundation (ISF grant J9K100), the National Science Foundation (Phy\#9602178), NATO (grant CR6.941058-1360/94), the Russian Academy of Science, Russian Foundation of Fundamental Investigations, the Russian Ministry of Science and Technology, U.S. Civilian Research \& Development Foundation, the U.S. Department of Energy (DOE grant DE-FG02-91ER40664 and DOE contract number DE-AC02-76CHO3000) and the U.S.-Israel Binational Science Foundation (BSF). 


\section{References}

[1] J. Wiss, Prepared for International School of Physics, 'Enrico Fermi': Heavy Flavor Physics - A Probe of Nature's Grand Design, Varenna, Italy, 8-18 Jul 1997

[2] J. S. Russ, Nucl. Phys. A 585 (1995) 39C.

[3] M. Y. Balats et al., ITEP-92-109

[4] Y. M. Goncharenko, O. A. Grachov, V. F. Kurshetsov, L. G. Landsberg, S. B. Nurushev and A. N. Vasiliev, IFVE-95-109

[5] J. Lach, FERMILAB-TM-2129

[6] J. Engelfried et al., Nucl. Instrum. Meth. A 433 (1999) 149.

[7] D. L. Adams et al., IFVE-91-100

[8] E. Dambik, D. Kline and R. West, FERMILAB-CONF-93-288 Presented at International Conference on Accelerator and Large Experimental Physics Control Systems (ICALEPCS 93), Berlin, Germany, 18-22 Oct 1993

[9] S. Zimmermann, V. H. Areti, G. W. Foster, U. Joshi and K. Treptow, FERMILAB-CONF-91-290 Presented at IEEE 1991 Nuclear Science Symp., Santa Fe, NM, Nov 5-9, 1991

[10] A. A. Lednev, IFVE-93-153

[11] A. A. Lednev, Nucl. Instrum. Meth. A 366 (1995) 292.

[12] D. Autiero et al., Nucl. Instrum. Meth. A 425 (1999) 188.

[13] K. Hagiwara et al. [Particle Data Group Collaboration], Phys. Rev. D 66 (2002) 010001.

[14] M. Kobayashi, Y. Prokoshkin, A. Singovsky and K. Takamatsu, Nucl. Instrum. Meth. A 345 (1994) 210.

[15] A. V. Inyakin, D. B. Kakauridze, A. A. Lednev, Y. D. Prokoshkin and A. V. Singovsky, Nucl. Instrum. Meth. 215 (1983) 103. 


\section{Tables}

Table 1: Specifications of the $S E L E X$ lead-glass calorimeters.

\begin{tabular}{|c|c|c|c|c|c|}
\hline & \multicolumn{2}{|c|}{ blocks } & \multirow{2}{*}{$\begin{array}{l}\text { z position } \\
(\mathrm{cm})\end{array}$} & \multicolumn{2}{|c|}{ transverse dimensions } \\
\hline & small & large & & total $\left(\mathrm{cm}^{2}\right)$ & hole $\left(\mathrm{cm}^{2}\right)$ \\
\hline Photon 1 & 576 & 54 & 526.6 & $136.9 \times 111$ & $42.5 \times 16.5$ \\
\hline Photon 2 & 540 & 186 & 3573.2 & $230.9 \times 110.6$ & $50.7 \times 41.2$ \\
\hline Photon 3 & 316 & & 5003.9 & $80 \times 61$ & $15.2 \times 7.6$ \\
\hline
\end{tabular}

Table 2: The chemical structure of lead-glass.

\begin{tabular}{|c|c|c|c|}
\hline & F8-00 & TF1-000 & TF101 \\
\hline $\mathrm{PbO}$ & $45.0 \%$ & $51.2 \%$ & $51.2 \%$ \\
\hline $\mathrm{SiO}_{2}$ & $42.8 \%$ & $41.3 \%$ & $41.5 \%$ \\
\hline $\mathrm{K}_{2} \mathrm{O}$ & $10.4 \%$ & $7.0 \%$ & $7.0 \%$ \\
\hline $\mathrm{Na}_{2} \mathrm{O}$ & $1.8 \%$ & - & - \\
\hline $\mathrm{As}_{2} \mathrm{O}_{3}$ & - & $0.5 \%$ & - \\
\hline $\mathrm{CeO}_{2}$ & - & - & $0.2 \%$ \\
\hline Refractive index, $\mathrm{n}_{e}$ & 1.62 & 1.65 & 1.65 \\
\hline
\end{tabular}

Table 3. Cookies.

\begin{tabular}{|c|c|c|c|c|c|c|}
\hline \multirow{3}{*}{ Photon } & \multirow{2}{*}{ PMT } & \multicolumn{4}{|c|}{ Material } & \multirow{2}{*}{ Qty. } \\
\cline { 3 - 6 } & & \multicolumn{2}{|c|}{ Plexiglas } & \multicolumn{2}{|c|}{ RTV615 } \\
\cline { 3 - 6 } & $\begin{array}{c}\text { O.D. } \\
(\mathrm{mm})\end{array}$ & $\begin{array}{c}\text { THKNS } \\
(\mathrm{mm})\end{array}$ & $\begin{array}{c}\text { O.D. } \\
(\mathrm{mm})\end{array}$ & $\begin{array}{c}\text { THKNS } \\
(\mathrm{mm})\end{array}$ & \\
\hline Photon 1 & FEU-110 & 75 & 30 & 75 & 4 & 54 \\
\hline & FEU-84-3 & & & 30 & 15 & 576 \\
\hline Photon 2 & FEU-110 & & & 75 & 4 & 186 \\
\hline & FEU-84-3 & & & 32 & 4 & 540 \\
\hline Photon 3 & FEU-84-3 & & & 27 & 10 & 316 \\
\hline
\end{tabular}


Table 4. $\pi^{0}$ calibration results.

\begin{tabular}{|c|c|c|c|c|}
\hline $\begin{array}{c}\text { Photon } \\
\text { combination }\end{array}$ & $\begin{array}{c}\pi^{0} \text { mass } \\
(\mathrm{MeV})\end{array}$ & $\begin{array}{c}\pi^{0} \text { width } \\
(\mathrm{MeV})\end{array}$ & $\begin{array}{c}\sigma(M) / M \\
(\%)\end{array}$ & $\begin{array}{c}\text { photon energy range } \\
(\mathrm{GeV})\end{array}$ \\
\hline 11 & $133.8 \pm 0.05$ & $9.1 \pm 0.1$ & $6.5 \pm 0.1$ & $2-10$ \\
12 & $135.5 \pm 0.1$ & $8.4 \pm 0.1$ & $6.2 \pm 0.1$ & \\
13 & $134.5 \pm 1.2$ & $8.6 \pm 0.1$ & $6.3 \pm 0.1$ & \\
22 & $135.2 \pm 0.1$ & $6.4 \pm 0.1$ & $4.7 \pm 0.4$ & $2-40$ \\
23 & $135.2 \pm 0.1$ & $6.9 \pm 0.1$ & $5.0 \pm 0.4$ & \\
33 & $134.6 \pm 0.1$ & $7.3 \pm 0.1$ & $5.3 \pm 0.4$ & $2-80$ \\
\hline
\end{tabular}




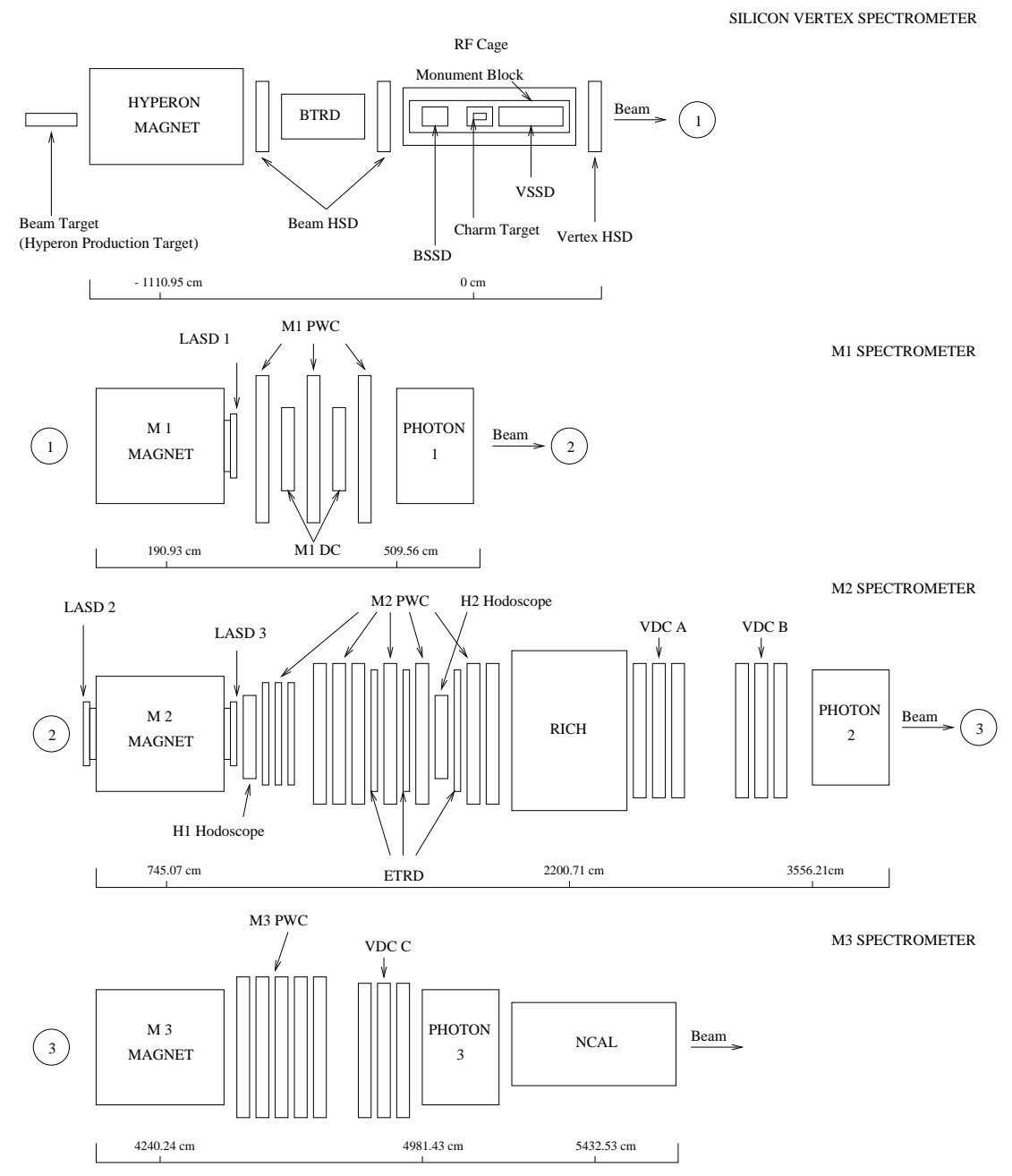

Fig. 1. Schematic diagram of the SELEX layout. See the text for the meaning of the labels.

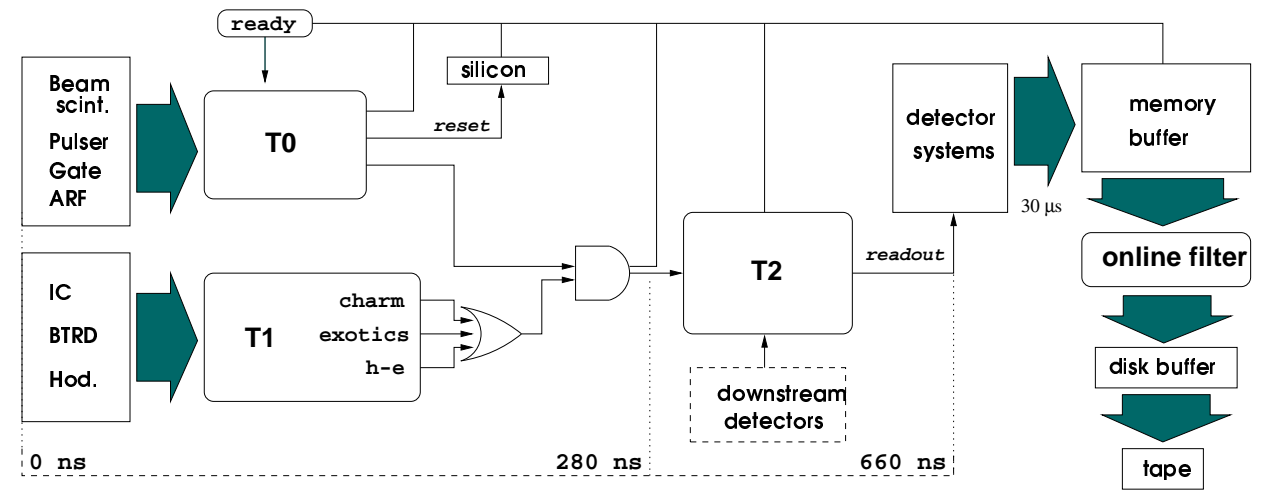

Fig. 2. A simplified scheme of the SELEX trigger. 


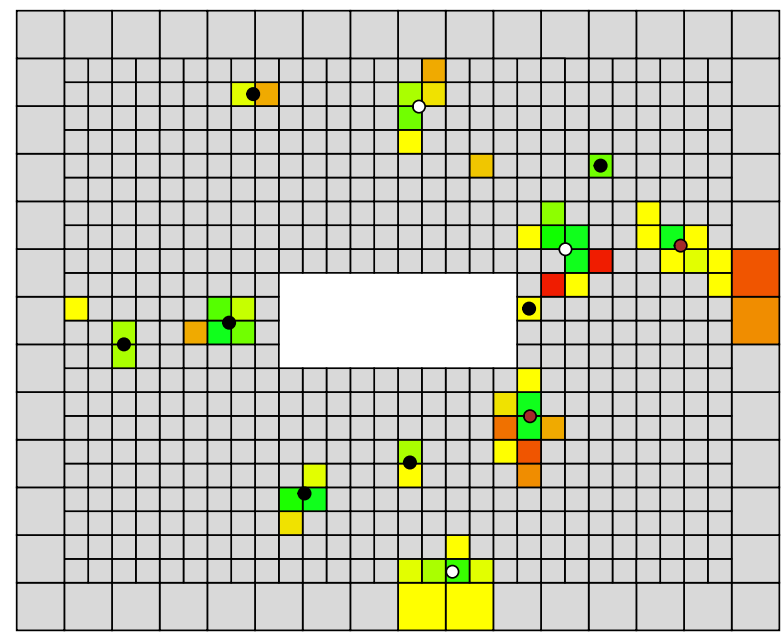

Fig. 3. Beam view of Photon 1. Colored blocks show the typical energy spread in a reconstructed event. Black dots are the coordinates of the photon clusters, white dots correspond to other neutral particles and shaded dots correspond to charged particles.

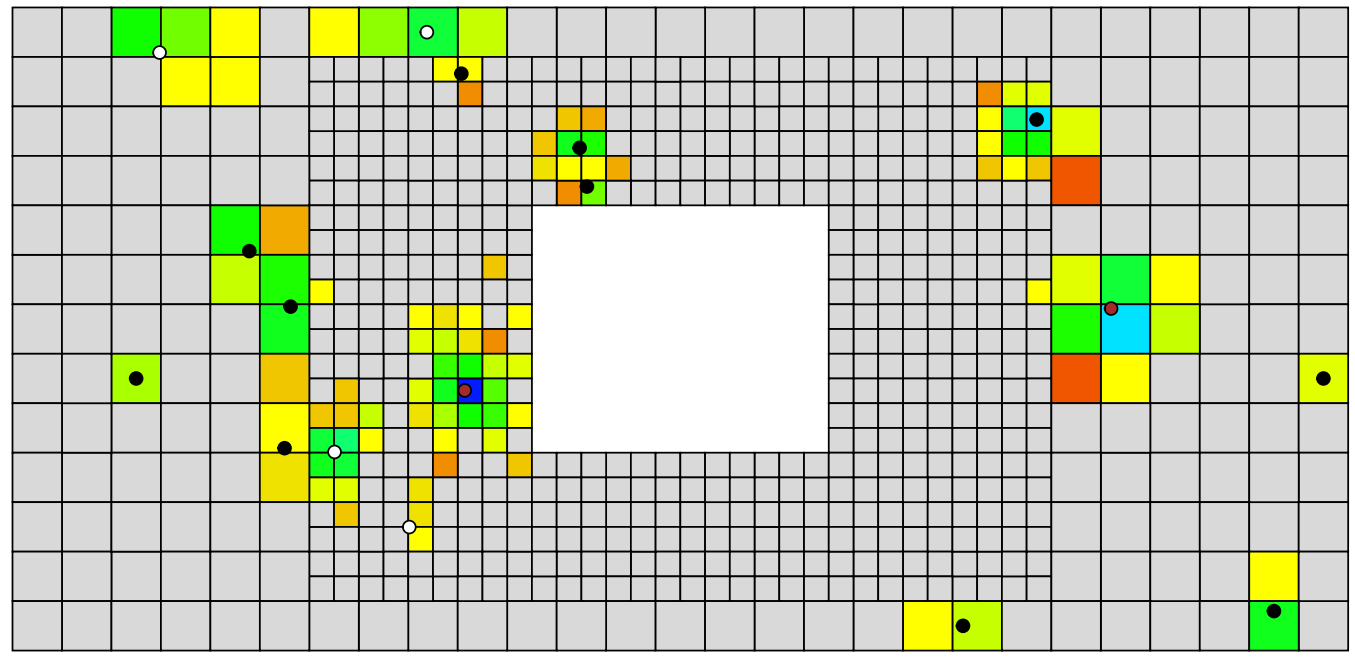

Fig. 4. Beam view of Photon 2. Colored blocks show the typical energy spread in a reconstructed event. Black dots are the coordinates of the photon clusters, white dots correspond to other neutral particles and shaded dots correspond to charged particles. 


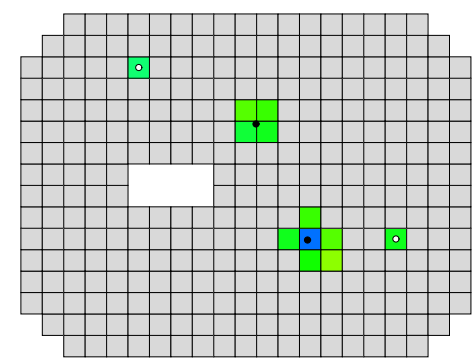

Fig. 5. Beam view of Photon 3. Colored blocks show the typical energy spread in a reconstructed event. Black dots are the coordinates of the photon clusters and white dots correspond to other neutral particles.

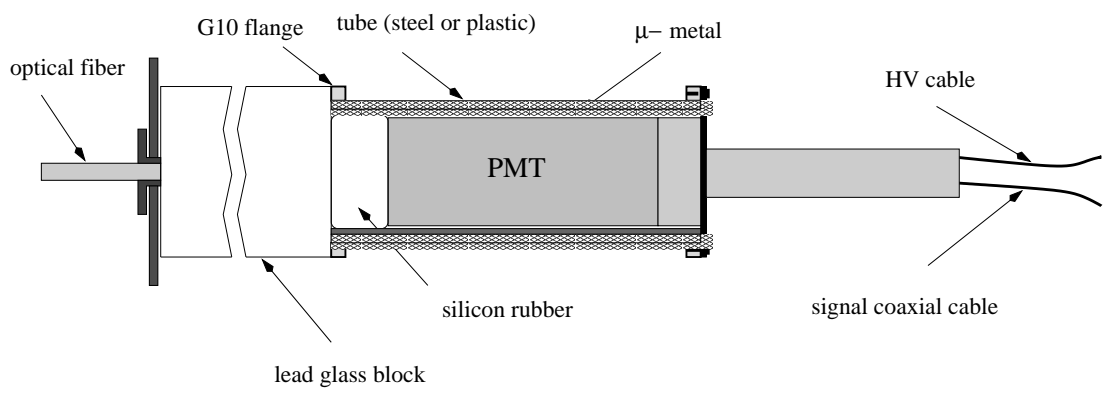

Fig. 6. Schematic layout of a lead-glass counter.

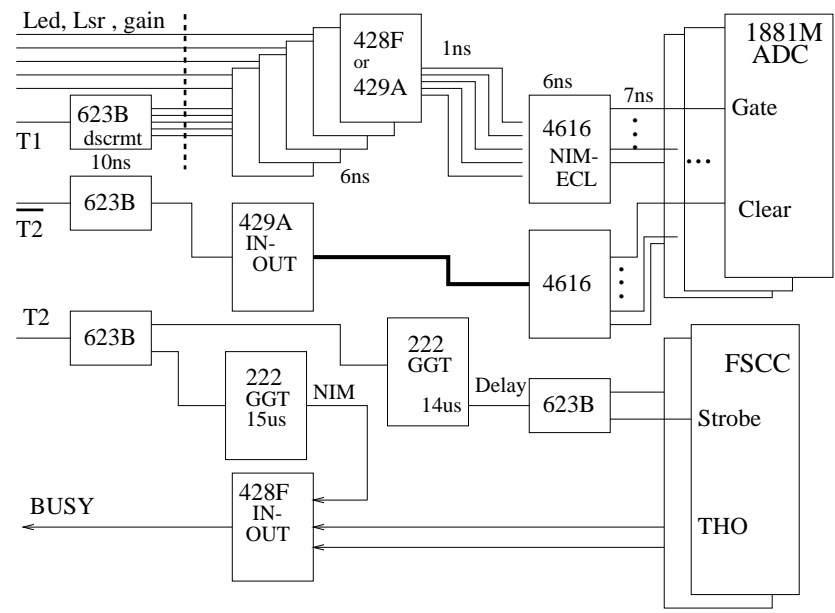

Fig. 7. Block diagram of the ADC local trigger logic. 


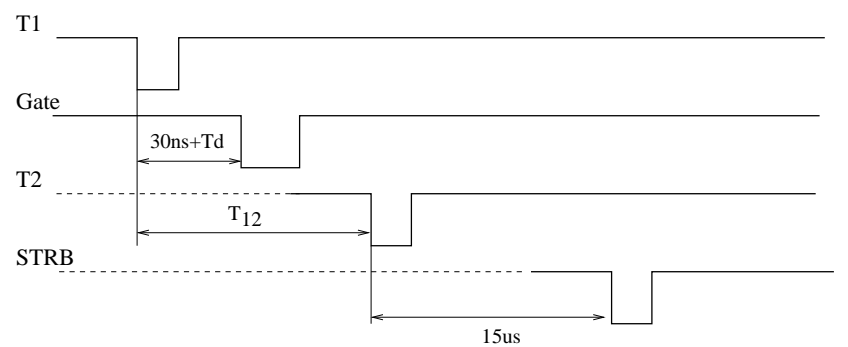

(A)

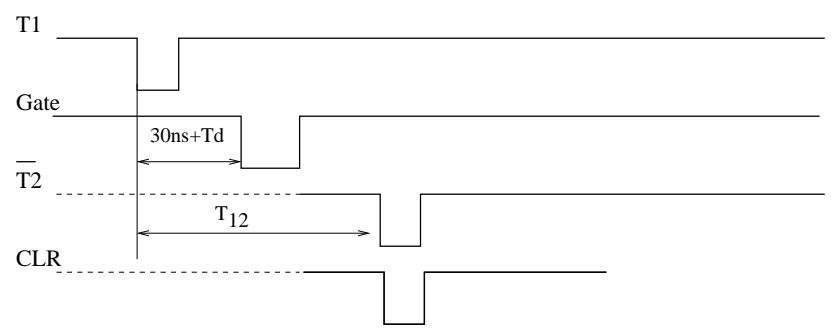

(B)

Fig. 8. ADC local trigger timing diagram. (A): $T 1$ with $T 2$. Gate and strobe to FSCC are generated, or event is accepted. (B) $T 1$ with $\bar{T} 2$. Gate and clear are generated or event is rejected.

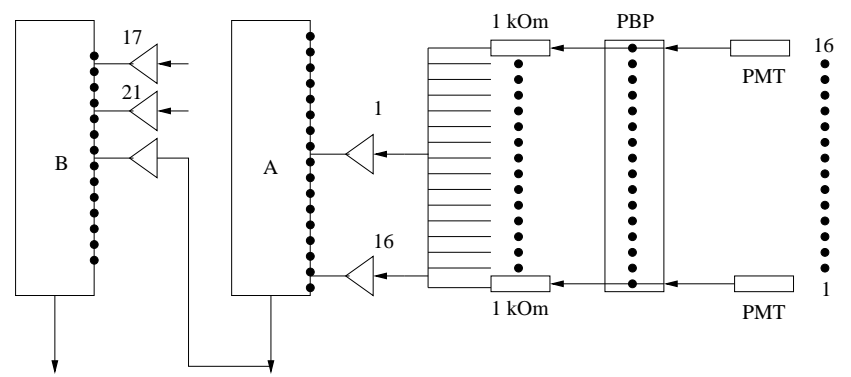

Fig. 9. A schematic view of Photon 3 trigger.

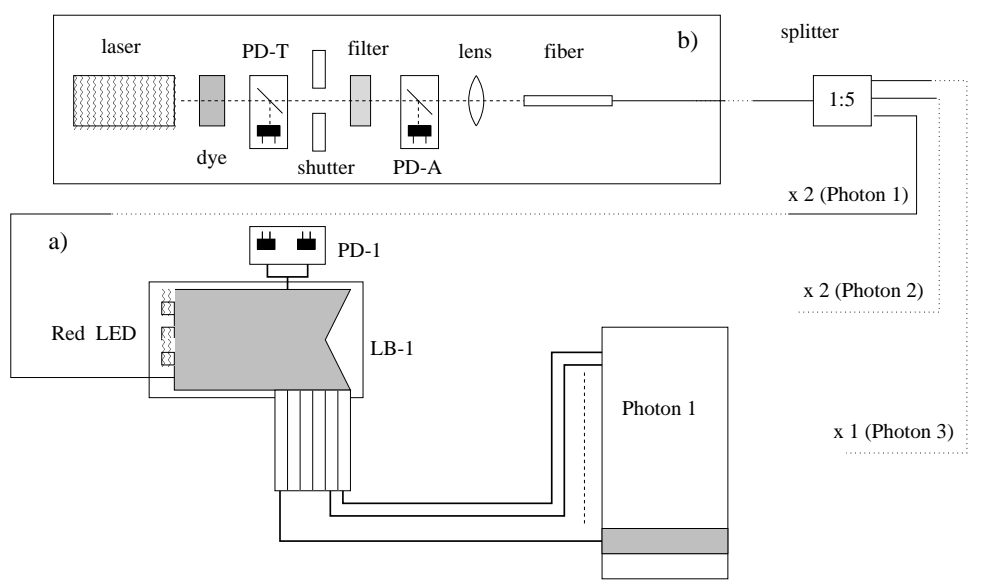

Fig. 10. A schematic view of the monitoring system. 


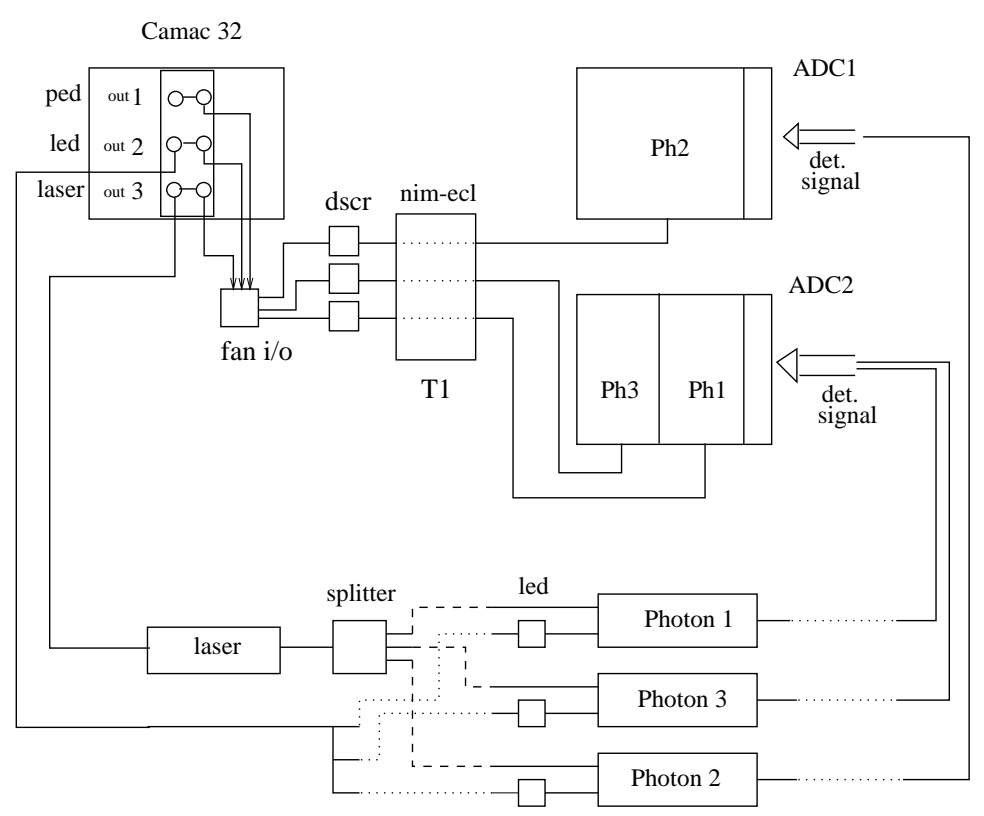

Fig. 11. A sketch of the readout electronics.
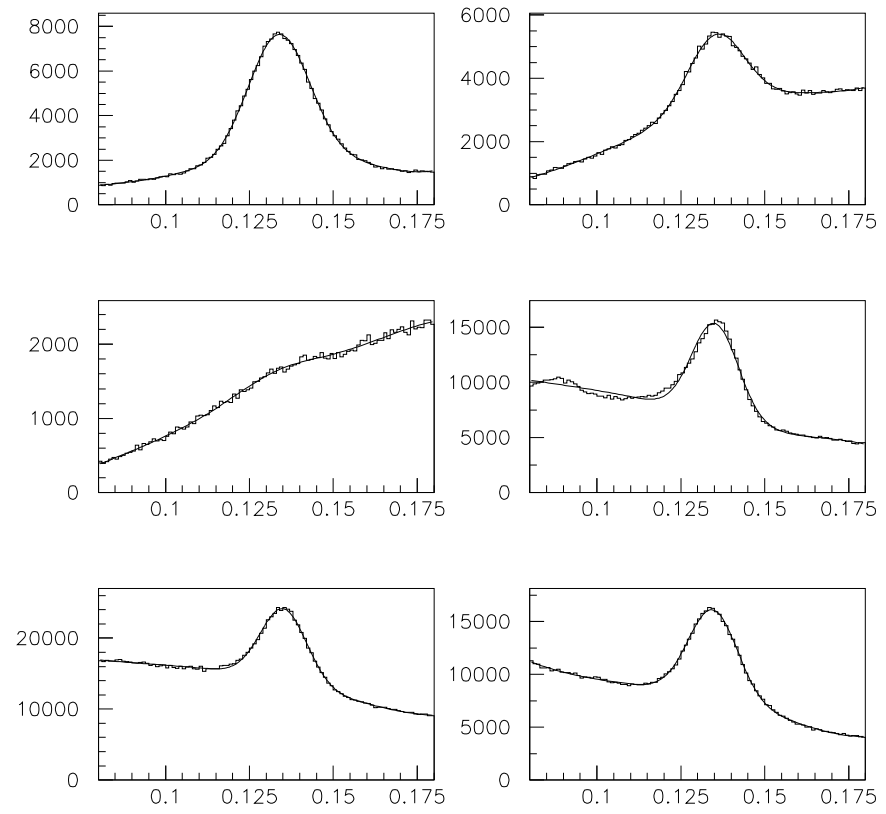

Fig. 12. The effective mass distribution for the $\gamma-\gamma$ system, for isolated photons with $E_{\gamma}>2 \mathrm{GeV}$. Top left: both photons hit Photon 1 (11). Top right: the first photon hits Photon 1 and the second hits Photon 2 (12). Middle left: the first photon hits Photon 1 and the second hits Photon 3 (13). Middle right: both photons hit Photon 2 (22). Bottom left: the first photon hits Photon 2 and the second hits Photon 3 (23). Bottom right: both photons hit Photon 3 (33). Fits are presented in Table 4. 

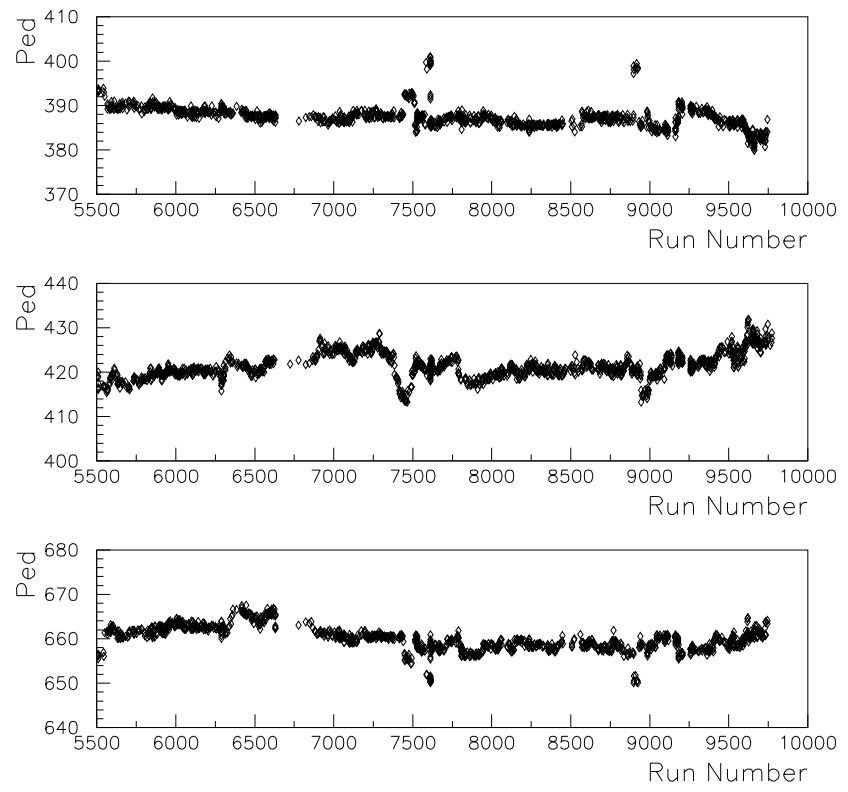

Fig. 13. ADC pedestal behavior for three typical counters (one from each of the Photon detectors) during data taking.
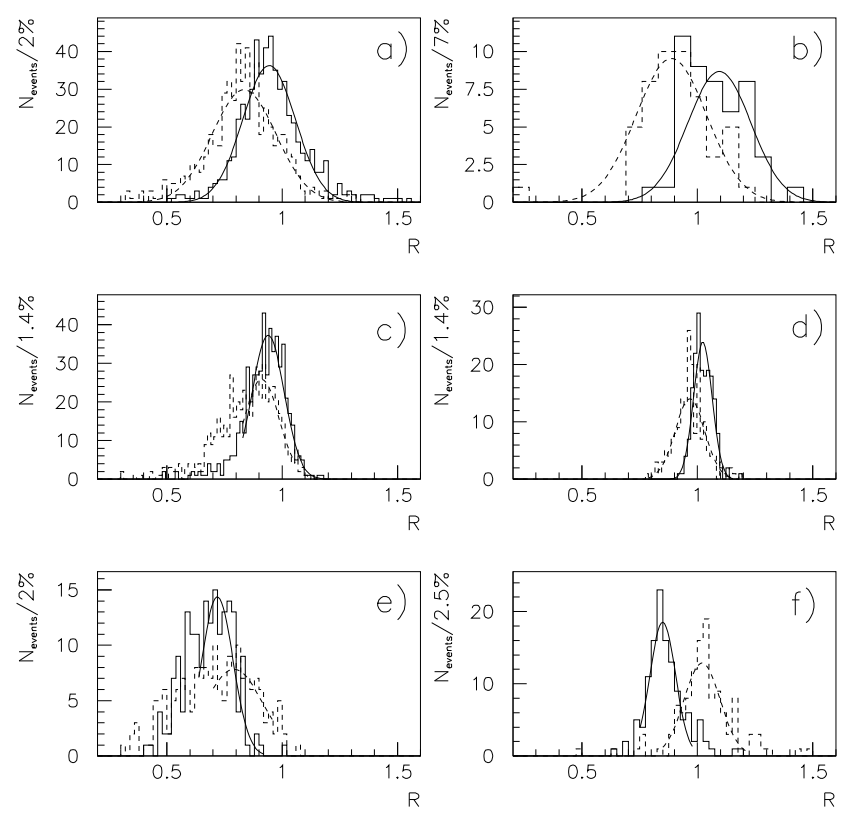

Fig. 14. Distributions of $R_{\text {corr }}$ (solid histogram) and $R_{\text {uncorr }}$ (dashed histogram) for (a) small and (b) big blocks of Photon 1, for (c) small and (d) big blocks of Photon 2 and for (e) ordinary and (f) radiation hard blocks of Photon 3. The Gaussian fits are shown by the curves. 

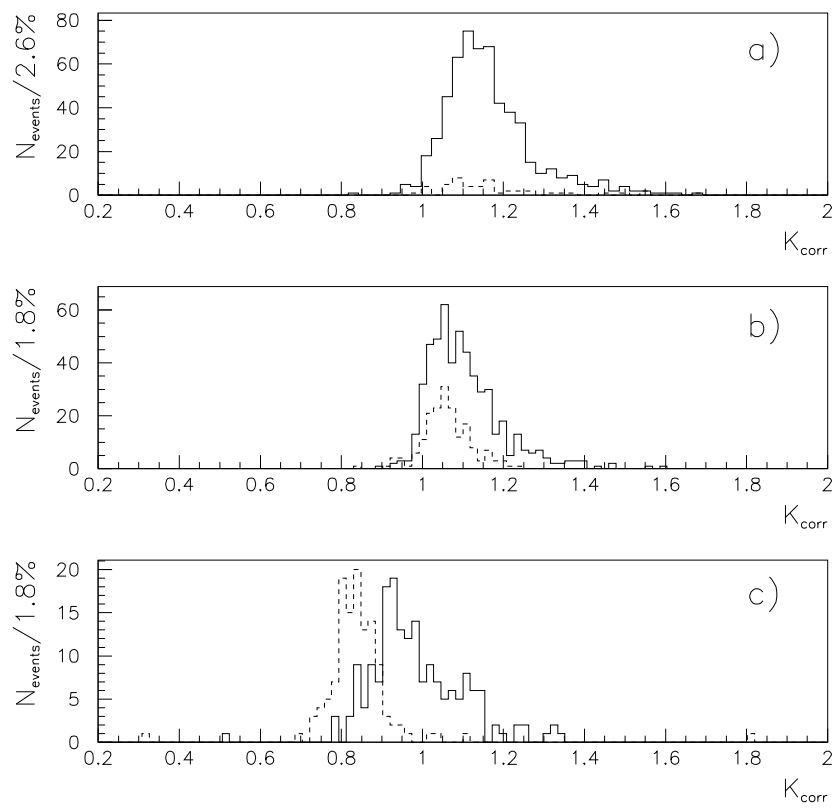

Fig. 15. Distribution of $K_{\text {corr }}$ : (a) - for small (dashed histogram) and big blocks (solid histogram) of Photon 1,(b) - for small (dashed histogram) and big blocks (solid histogram) of Photon 2 and (c) - for ordinary (dashed histogram) and radiation hard (solid histogram) of Photon 3.

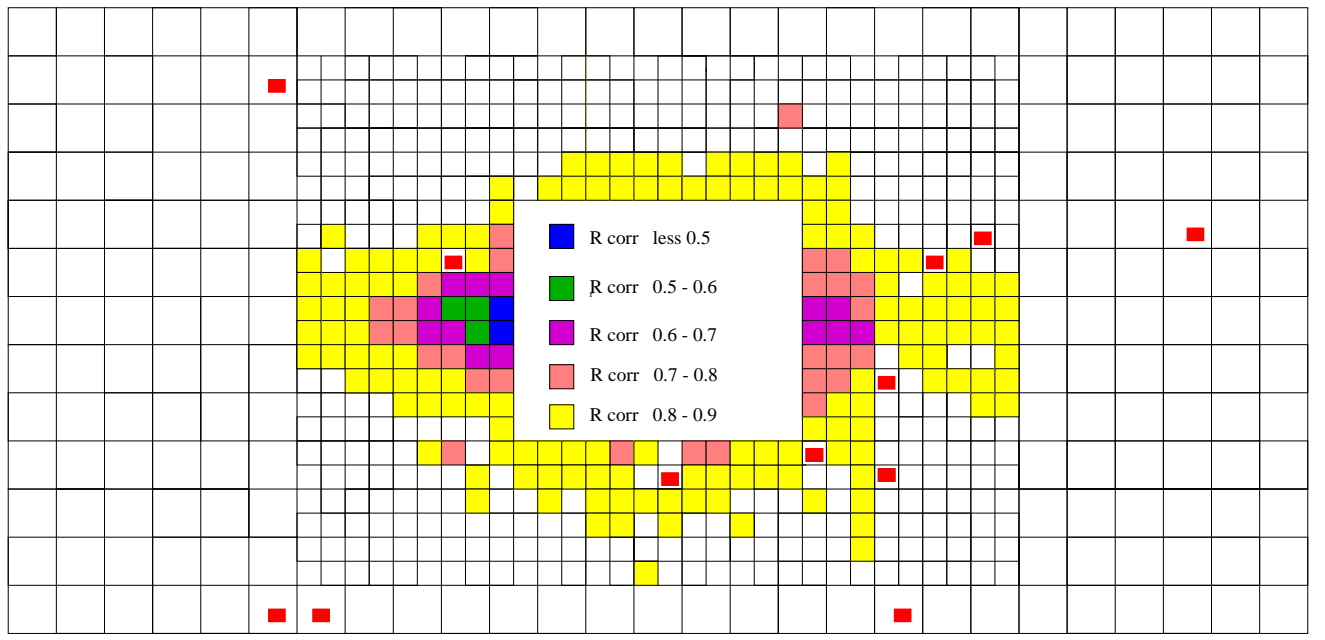

Fig. 16. The graphical mapping of $R_{\text {corr }}$ for some Photon 2 radiation-damaged counters. Counters with the same value of $R_{\text {corr }}$ are shown with the same color. Small hatched rectangles mark the blocks with replaced channels which were excluded from the analysis. 


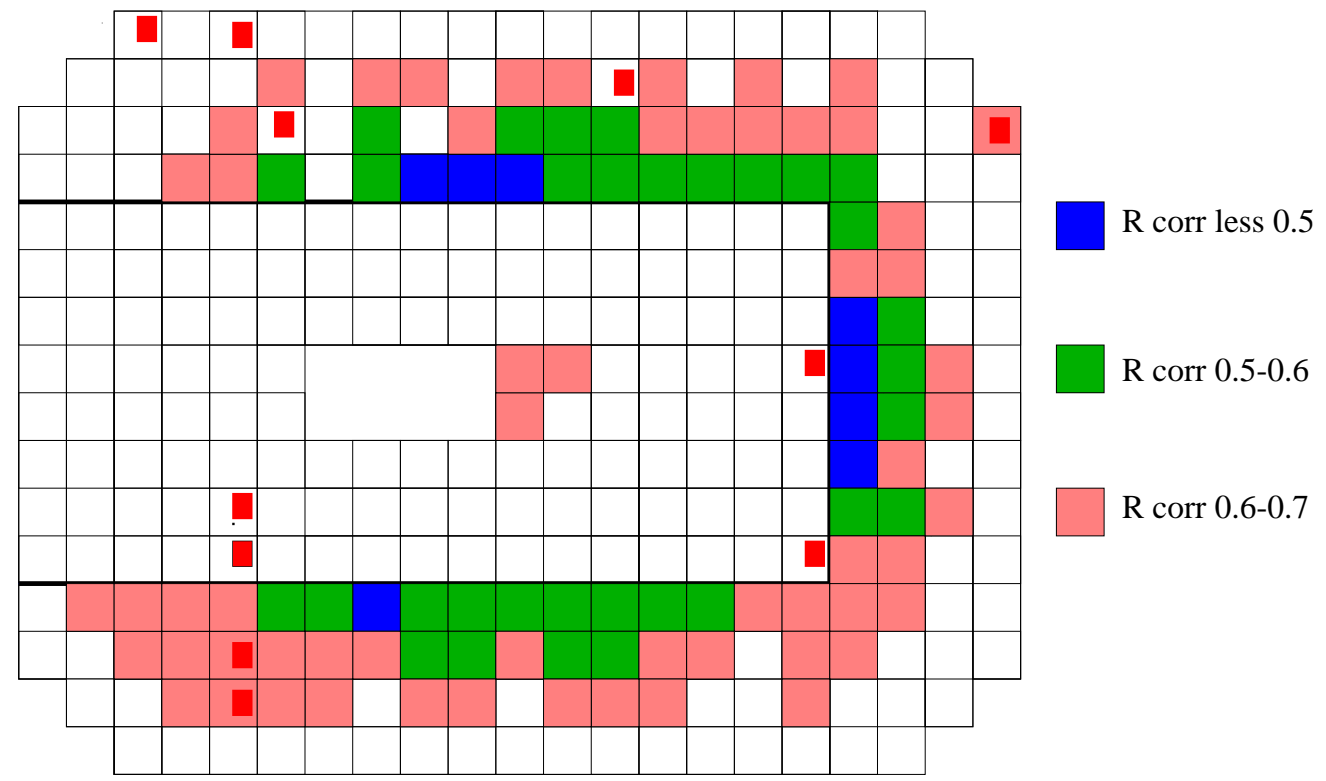

Fig. 17. The graphical mapping of $R_{\text {corr }}$ for some Photon 3 radiation damaged counters. Counters with the same value of $R_{\text {corr }}$ are shown with the same color. Small hatched rectangles mark the blocks with replaced channels which were excluded from the analysis.
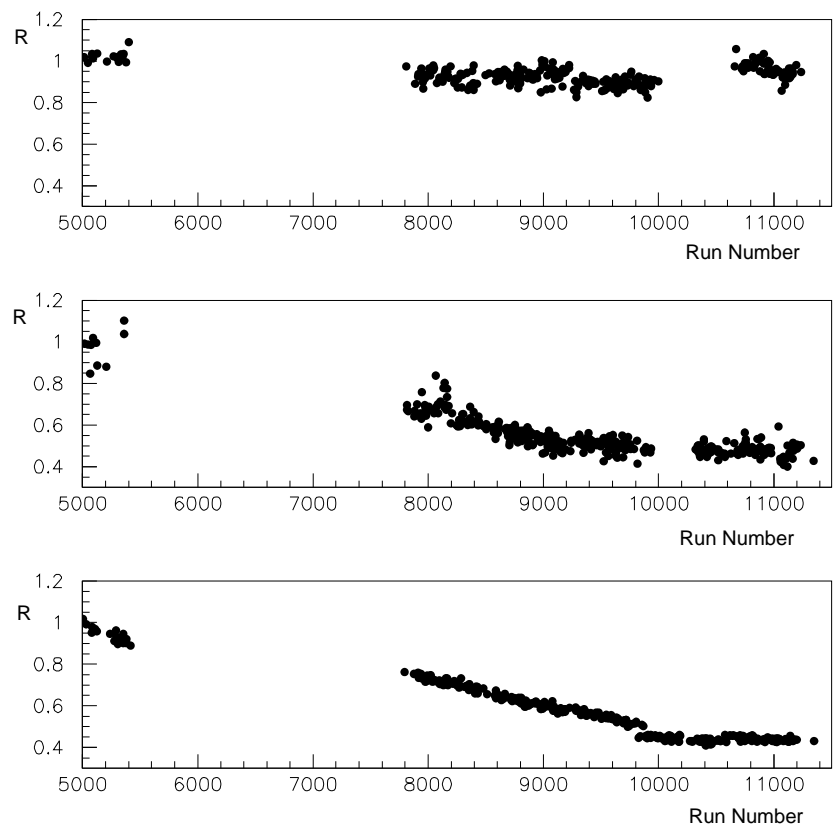

Fig. 18. The long-term variation of $R_{\text {corr }}$ in time for typical counters from Photon 1 (top), for the damaged counters from Photon 2 (middle) and for the damaged counters from Photon 3 (bottom). 


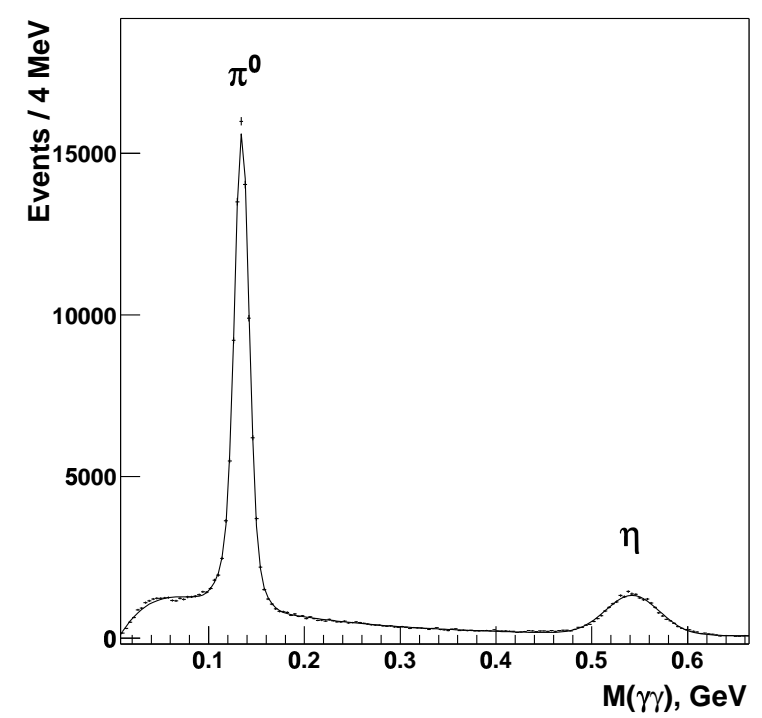

Fig. 19. The effective mass distribution for the $\gamma \gamma$ system $\left(\pi^{-}\right.$beam, Exclusive trigger, $\left.E_{\gamma}>2 \mathrm{GeV}, N_{\gamma}=2, E_{\gamma \gamma}>10 \mathrm{GeV}\right)$. The mass resolution $\sigma(M) / M$ is $6 \%$ for the $\pi^{0}$ and $5 \%$ for the $\eta$.

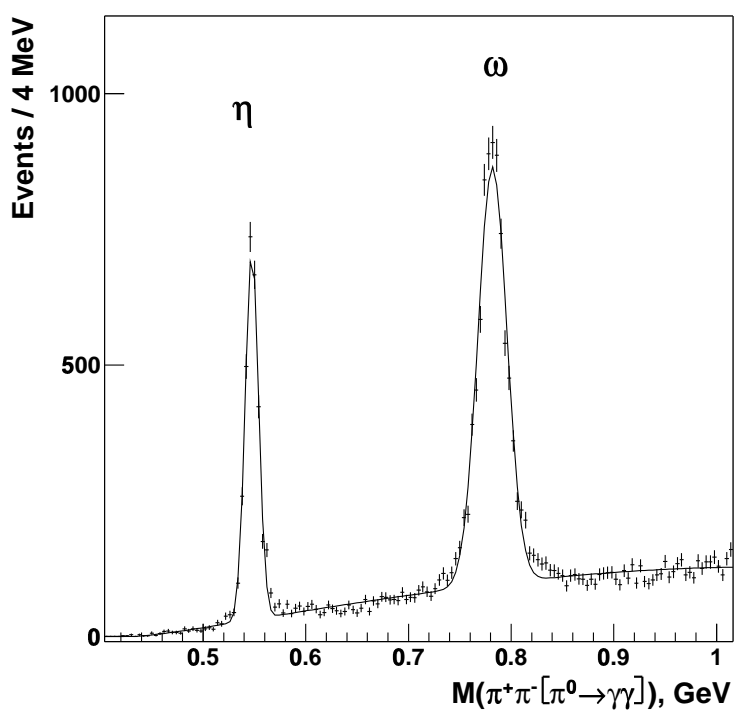

Fig. 20. The effective mass distribution for the $\pi^{+} \pi^{-} \pi^{0}$ system $\left(\Sigma^{-}\right.$beam, Exclusive trigger, $\left.E_{\gamma}>2 \mathrm{GeV}, N_{\gamma}=2, E_{\pi^{0}}>10 \mathrm{GeV}\right)$. The mass resolution $\sigma(M) / M$ is $1.2 \%$ for the $\eta$ and $1.8 \%$ for the $\omega$. 


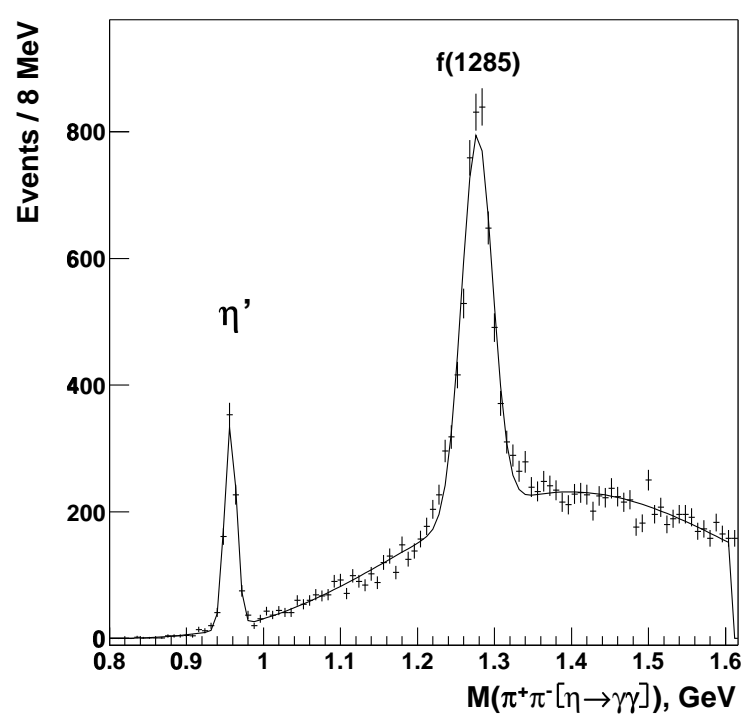

Fig. 21. The effective mass distribution for the $\pi^{+} \pi^{-} \eta$ system (Exclusive trigger, $\left.E_{\gamma}>2 \mathrm{GeV}, N_{\gamma}=2, E_{\eta}>10 \mathrm{GeV}\right)$. The mass resolution $\sigma(M) / M$ is $0.8 \%$ for the $\eta^{\prime}$ and $1.35 \%$ for the $f$.

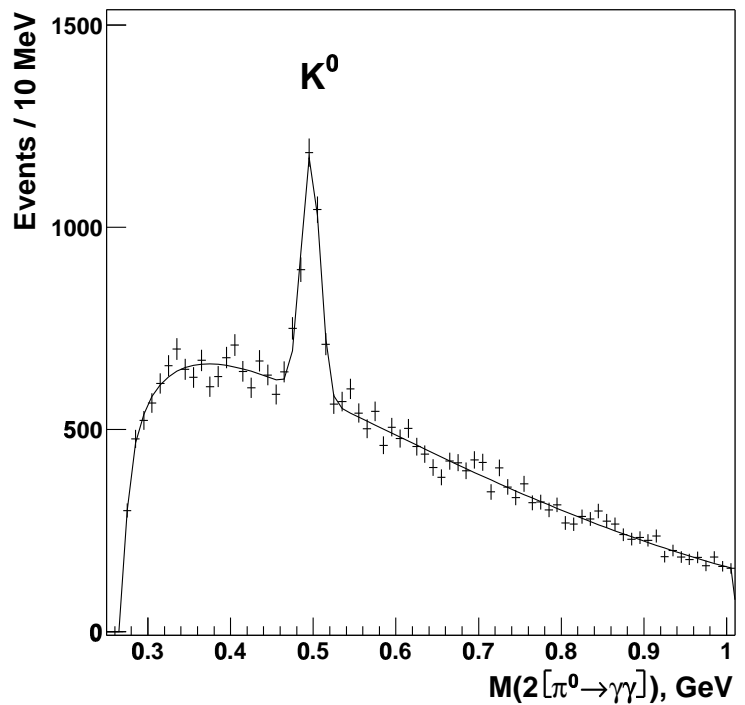

Fig. 22. The effective mass distribution for the $2 \pi^{0}$ system $\left(\Sigma^{-}\right.$beam, Exclusive trigger, $\left.E_{\gamma}>4 \mathrm{GeV}, N_{\pi^{0}} \leq 4, E_{\pi^{0}}>30 \mathrm{GeV}\right)$. The mass resolution $\sigma(M) / M$ for the $K^{0}$ is $2.2 \%$. 


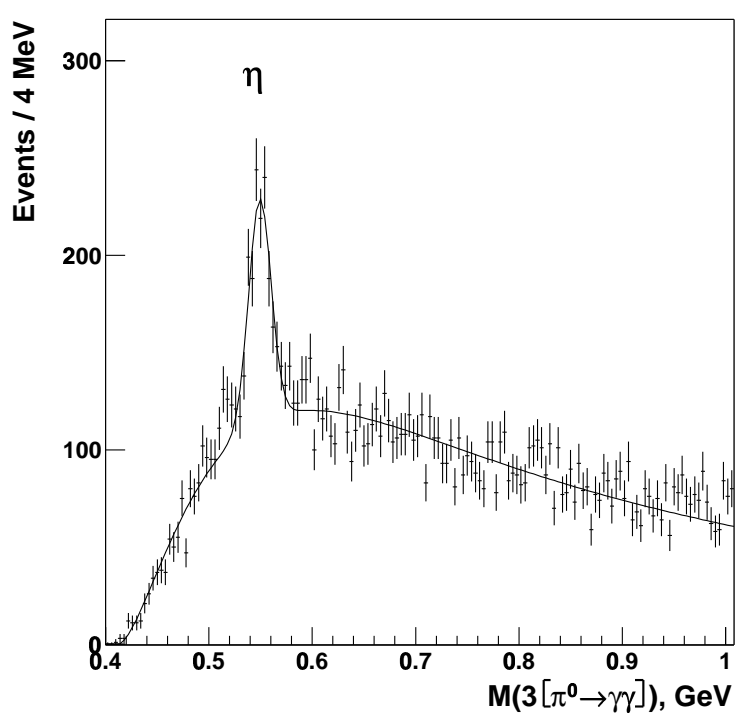

Fig. 23. The effective mass distribution for the $3 \pi^{0}$ system $\left(\Sigma^{-}\right.$beam, Exclusive trigger, $\left.E_{\gamma}>5 \mathrm{GeV}, N_{\gamma}=6, E_{\pi^{0}}>20 \mathrm{GeV}\right)$. The mass resolution $\sigma(M) / M$ for the $\eta$ is $1.9 \%$.

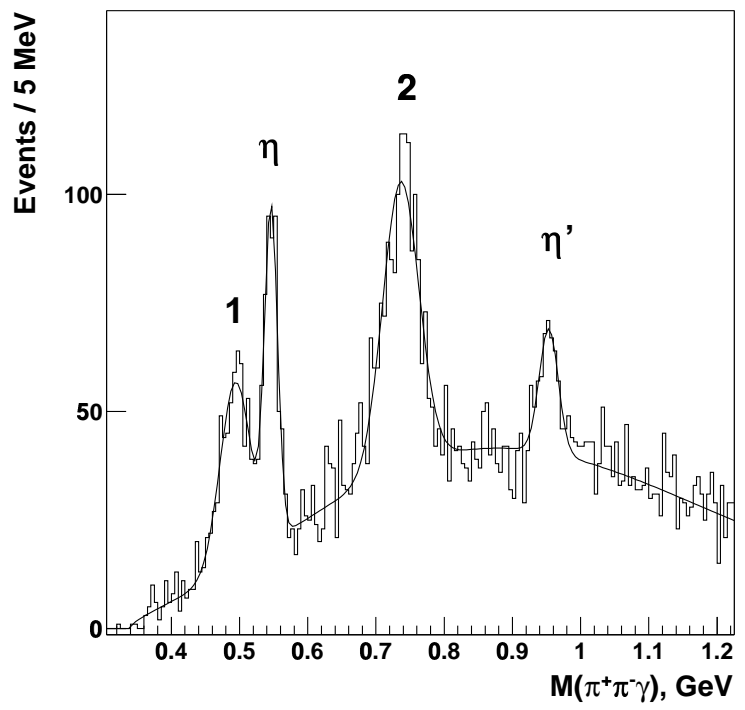

Fig. 24. The effective mass distribution for the $\pi^{+} \pi^{-} \gamma$ system $\left(\Sigma^{-}\right.$beam, Exclusive trigger). 1 is a "false" peak from the decay process $\eta \rightarrow \pi^{+} \pi^{-} \pi^{0}$ with one photon missing. 2 is a "false" peak from the decay process $\omega \rightarrow \pi^{+} \pi^{-} \pi^{0}$ with one photon missing. False peaks are shifted to lower values of mass. 


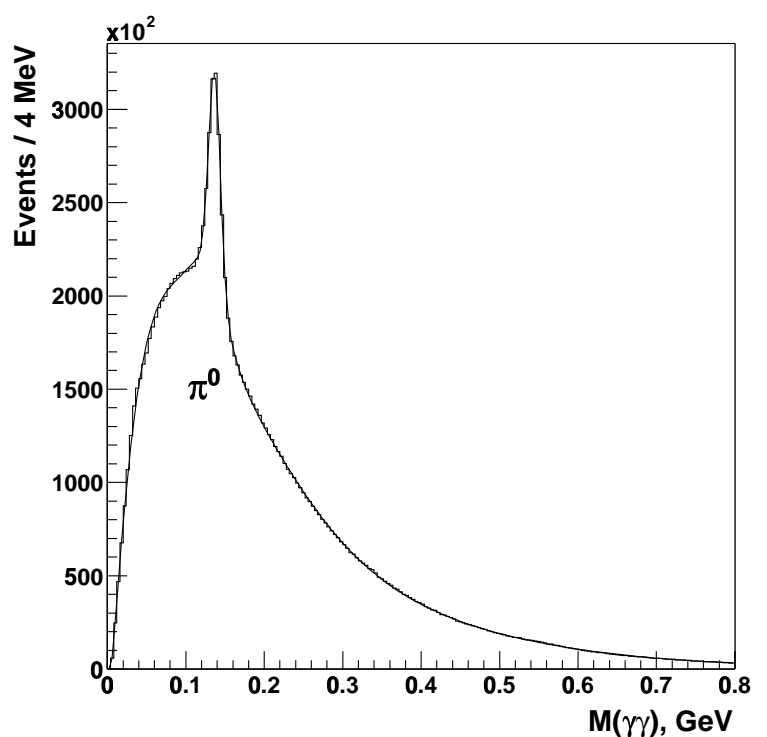

Fig. 25. The effective mass distribution for the $\gamma \gamma$ system (Charm trigger, $N_{\gamma} \leq 10$, $\left.E_{\gamma}>2 \mathrm{GeV}, E_{\gamma \gamma}>10 \mathrm{GeV}\right)$. The $\pi^{0}$ mass resolution $\sigma(M) / M$ is $6 \%$.

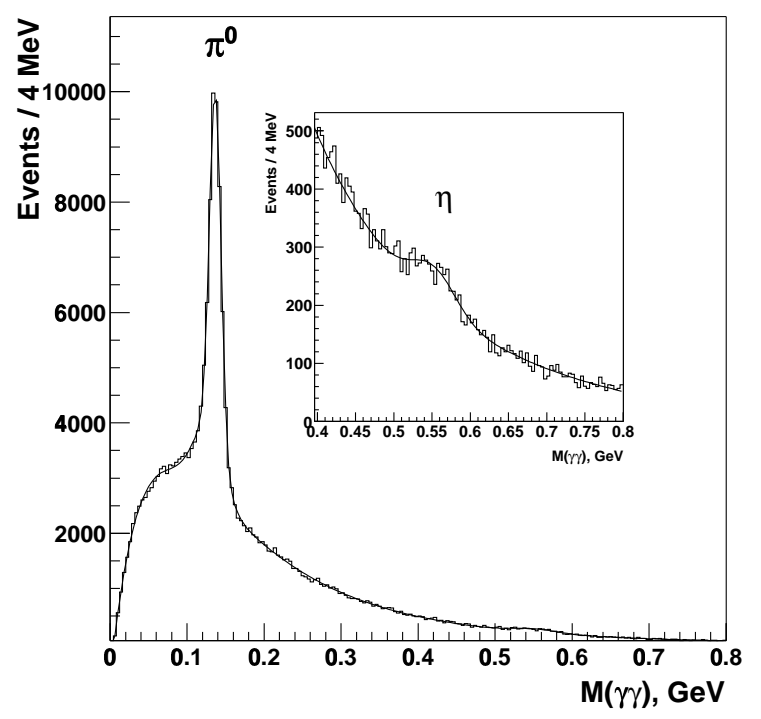

Fig. 26. The effective mass distribution for the $\gamma \gamma$ system (charm trigger, $N_{\gamma}=2$, $\left.E_{\gamma}>2 \mathrm{GeV}, E_{\gamma \gamma}>10 \mathrm{GeV}\right)$. The $\pi^{0}$ mass resolution $\sigma(M) / M$ is $7 \%$ and the $\eta$ mass resolution $\sigma(M) / M$ is $5 \%$. 


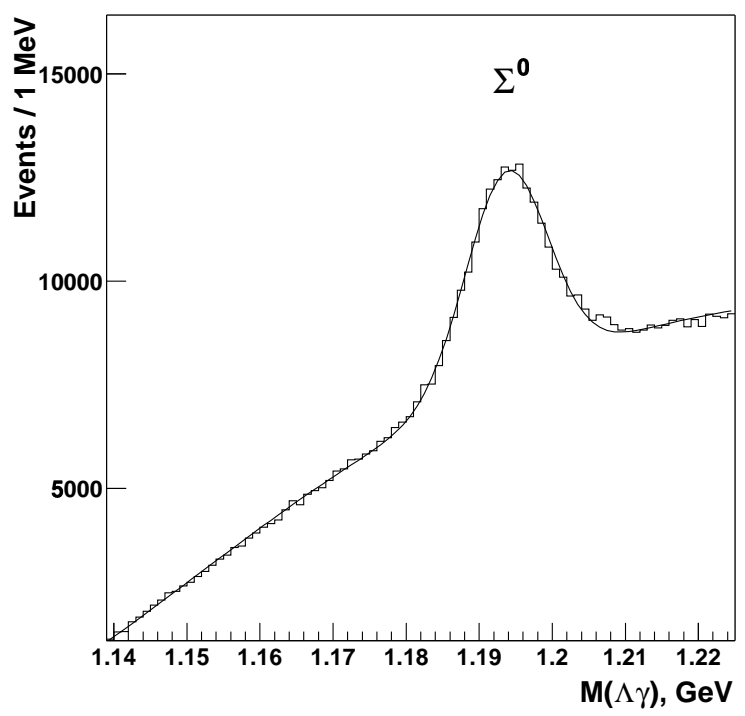

Fig. 27. The effective mass distribution for the $\Lambda \gamma$ system (Charm trigger with $\left.E_{\gamma}>6 \mathrm{GeV}\right)$. The $\Sigma^{0}$ mass resolution $\sigma(M) / M$ is $0.46 \%$.

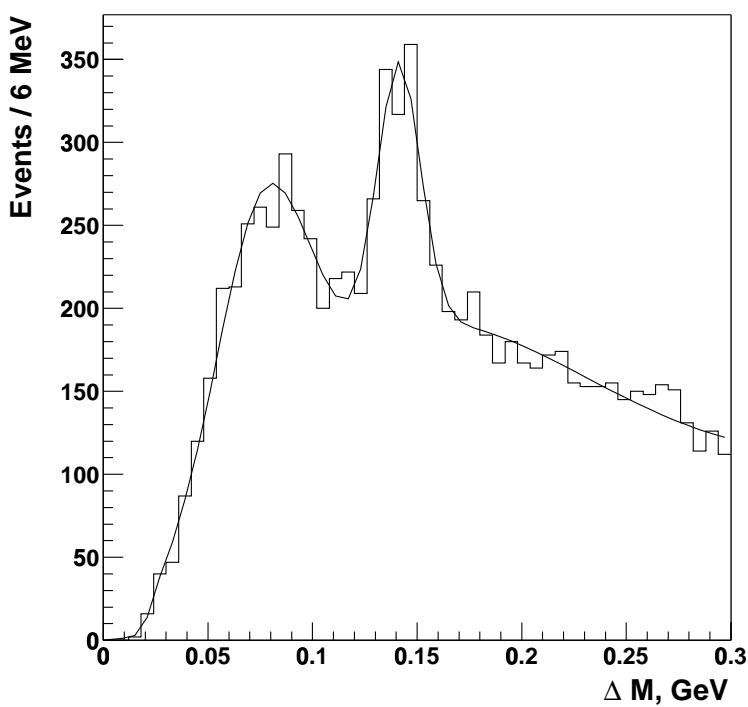

Fig. 28. The $\Delta M_{\gamma}=M\left(D^{0} \gamma\right)-M\left(D^{0}\right)$ distribution (Charm trigger with $\left.E_{\gamma}>5 \mathrm{GeV}\right)$. The mass resolution $\sigma(\Delta M)$ is $10.3 \mathrm{MeV}$. 


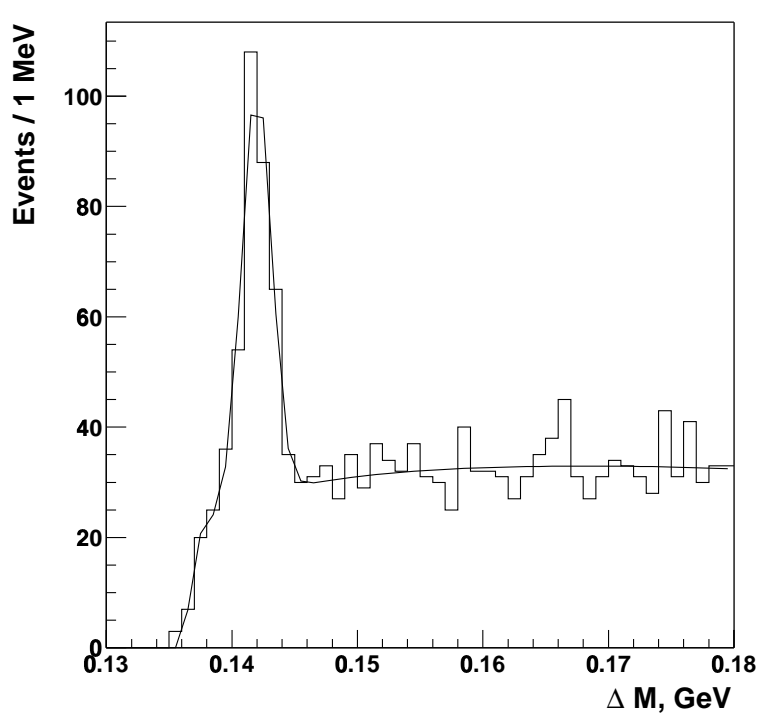

Fig. 29. The $\Delta M_{\pi}^{0}=M\left(D^{0} \pi^{0}\right)-M\left(D^{0}\right)$ distribution (Charm trigger with $\left.E_{\gamma 1,2}>3 \mathrm{GeV}\right)$. The mass resolution $\sigma(\Delta M)$ is $1.2 \mathrm{MeV}$. 


\section{List of Figures}

1 Schematic diagram of the SELEX layout. See the text for the meaning of the labels.

2 A simplified scheme of the SELEX trigger.

3 Beam view of Photon 1. Colored blocks show the typical energy spread in a reconstructed event. Black dots are the coordinates of the photon clusters, white dots correspond to other neutral particles and shaded dots correspond to charged particles.

4 Beam view of Photon 2. Colored blocks show the typical energy spread in a reconstructed event. Black dots are the coordinates of the photon clusters, white dots correspond to other neutral particles and shaded dots correspond to charged particles.

5 Beam view of Photon 3. Colored blocks show the typical energy spread in a reconstructed event. Black dots are the coordinates of the photon clusters and white dots correspond to other neutral particles.

$6 \quad$ Schematic layout of a lead-glass counter.

$7 \quad$ Block diagram of the ADC local trigger logic.

8 ADC local trigger timing diagram. (A): $T 1$ with $T 2$. Gate and strobe to FSCC are generated, or event is accepted. (B) $T 1$ with $\bar{T} 2$. Gate and clear are generated or event is rejected.

9 A schematic view of Photon 3 trigger.

10 A schematic view of the monitoring system. 
12 The effective mass distribution for the $\gamma-\gamma$ system, for isolated photons with $E_{\gamma}>2 \mathrm{GeV}$. Top left: both photons hit Photon 1 (11). Top right: the first photon hits Photon 1 and the second hits Photon 2 (12). Middle left: the first photon hits Photon 1 and the second hits Photon 3 (13). Middle right: both photons hit Photon 2 (22). Bottom left: the first photon hits Photon 2 and the second hits Photon 3 (23). Bottom right: both photons hit Photon 3 (33). Fits are presented in Table 4.

13 ADC pedestal behavior for three typical counters (one from each of the Photon detectors) during data taking.

14 Distributions of $R_{\text {corr }}$ (solid histogram) and $R_{\text {uncorr }}$ (dashed histogram) for (a) small and (b) big blocks of Photon 1, for (c) small and (d) big blocks of Photon 2 and for (e) ordinary and (f) radiation hard blocks of Photon 3. The Gaussian fits are shown by the curves.

15 Distribution of $K_{\text {corr }}$ : (a) - for small (dashed histogram) and big blocks (solid histogram) of Photon 1,(b) - for small (dashed histogram) and big blocks (solid histogram) of Photon 2 and (c) - for ordinary (dashed histogram) and radiation hard (solid histogram) of Photon 3.

16 The graphical mapping of $R_{\text {corr }}$ for some Photon 2 radiationdamaged counters. Counters with the same value of $R_{\text {corr }}$ are shown with the same color. Small hatched rectangles mark the blocks with replaced channels which were excluded from the analysis.

17 The graphical mapping of $R_{\text {corr }}$ for some Photon 3 radiation damaged counters. Counters with the same value of $R_{\text {corr }}$ are shown with the same color. Small hatched rectangles mark the blocks with replaced channels which were excluded from the analysis.

18 The long-term variation of $R_{\text {corr }}$ in time for typical counters from Photon 1 (top), for the damaged counters from Photon 2 (middle) and for the damaged counters from Photon 3 (bottom).

19 The effective mass distribution for the $\gamma \gamma$ system $\left(\pi^{-}\right.$beam, Exclusive trigger, $\left.E_{\gamma}>2 \mathrm{GeV}, N_{\gamma}=2, E_{\gamma \gamma}>10 \mathrm{GeV}\right)$. The mass resolution $\sigma(M) / M$ is $6 \%$ for the $\pi^{0}$ and $5 \%$ for the $\eta$. 
20 The effective mass distribution for the $\pi^{+} \pi^{-} \pi^{0}$ system ( $\Sigma^{-}$beam, Exclusive trigger, $E_{\gamma}>2 \mathrm{GeV}, N_{\gamma}=2$, $\left.E_{\pi^{0}}>10 \mathrm{GeV}\right)$. The mass resolution $\sigma(M) / M$ is $1.2 \%$ for the $\eta$ and $1.8 \%$ for the $\omega$.

21 The effective mass distribution for the $\pi^{+} \pi^{-} \eta$ system (Exclusive trigger, $E_{\gamma}>2 \mathrm{GeV}, N_{\gamma}=2, E_{\eta}>10 \mathrm{GeV}$ ). The mass resolution $\sigma(M) / M$ is $0.8 \%$ for the $\eta^{\prime}$ and $1.35 \%$ for the $f$.

22 The effective mass distribution for the $2 \pi^{0}$ system $\left(\Sigma^{-}\right.$beam, Exclusive trigger, $\left.E_{\gamma}>4 \mathrm{GeV}, N_{\pi^{0}} \leq 4, E_{\pi^{0}}>30 \mathrm{GeV}\right)$. The mass resolution $\sigma(M) / M$ for the $K^{0}$ is $2.2 \%$.

23 The effective mass distribution for the $3 \pi^{0}$ system $\left(\Sigma^{-}\right.$beam, Exclusive trigger, $\left.E_{\gamma}>5 \mathrm{GeV}, N_{\gamma}=6, E_{\pi^{0}}>20 \mathrm{GeV}\right)$. The mass resolution $\sigma(M) / M$ for the $\eta$ is $1.9 \%$.

24 The effective mass distribution for the $\pi^{+} \pi^{-} \gamma$ system $\left(\Sigma^{-}\right.$ beam, Exclusive trigger). 1 is a "false" peak from the decay process $\eta \rightarrow \pi^{+} \pi^{-} \pi^{0}$ with one photon missing. 2 is a "false" peak from the decay process $\omega \rightarrow \pi^{+} \pi^{-} \pi^{0}$ with one photon missing. False peaks are shifted to lower values of mass.

25 The effective mass distribution for the $\gamma \gamma$ system (Charm trigger, $\left.N_{\gamma} \leq 10, E_{\gamma}>2 \mathrm{GeV}, E_{\gamma \gamma}>10 \mathrm{GeV}\right)$. The $\pi^{0}$ mass resolution $\sigma(M) / M$ is $6 \%$.

26 The effective mass distribution for the $\gamma \gamma$ system (charm trigger, $\left.N_{\gamma}=2, E_{\gamma}>2 \mathrm{GeV}, E_{\gamma \gamma}>10 \mathrm{GeV}\right)$. The $\pi^{0}$ mass resolution $\sigma(M) / M$ is $7 \%$ and the $\eta$ mass resolution $\sigma(M) / M$ is $5 \%$.

27 The effective mass distribution for the $\Lambda \gamma$ system (Charm trigger with $\left.E_{\gamma}>6 \mathrm{GeV}\right)$. The $\Sigma^{0}$ mass resolution $\sigma(M) / M$ is $0.46 \%$.

28 The $\Delta M_{\gamma}=M\left(D^{0} \gamma\right)-M\left(D^{0}\right)$ distribution (Charm trigger with $\left.E_{\gamma}>5 \mathrm{GeV}\right)$. The mass resolution $\sigma(\Delta M)$ is $10.3 \mathrm{MeV}$.

29 The $\Delta M_{\pi}^{0}=M\left(D^{0} \pi^{0}\right)-M\left(D^{0}\right)$ distribution (Charm trigger with $\left.E_{\gamma 1,2}>3 \mathrm{GeV}\right)$. The mass resolution $\sigma(\Delta M)$ is $1.2 \mathrm{MeV}$. 